\title{
Critical Review of Transcutaneous Vagus Nerve Stimulation: Challenges for Translation to Clinical Practice
}

OPEN ACCESS

Edited by:

Tianruo Guo,

University of New South Wales,

Australia

Reviewed by:

Peijing Rong

China Academy of Chinese Medical

Sciences, China

Xiaohong Sui,

Shanghai Jiao Tong University, China

${ }^{*}$ Correspondence:

Tatiana Kameneva

tkameneva@swin.edu.au

†These authors have contributed equally to this work

Specialty section:

This article was submitted to

Neural Technology,

a section of the journal

Frontiers in Neuroscience

Received: 02 September 2019 Accepted: 12 March 2020

Published: 28 April 2020

Citation:

Yap JYY, Keatch C, Lambert E,

Woods W, Stoddart PR and Kameneva T (2020) Critical Review of

Transcutaneous Vagus Nerve

Stimulation: Challenges for Translation to Clinical Practice.

Front. Neurosci. 14:284. doi: 10.3389/fnins.2020.00284

\author{
Jonathan Y. Y. Yap ${ }^{1 \dagger}$, Charlotte Keatch ${ }^{2 \dagger}$, Elisabeth Lambert ${ }^{3,4}$, Will Woods ${ }^{3}$, \\ Paul R. Stoddart ${ }^{1,2}$ and Tatiana Kameneva ${ }^{2,4,5 *}$
}

${ }^{1}$ ARC Training Centre in Biodevices, Swinburne University of Technology, Hawthorn, VIC, Australia, ${ }^{2}$ Faculty of Science, Engineering and Technology, Swinburne University of Technology, Hawthorn, VIC, Australia, ${ }^{3}$ School of Health Sciences, Swinburne University of Technology, Hawthorn, VIC, Australia, ${ }^{4}$ Verson Health Innovation Research Institute, Swinburne University of Technology, Hawthorn, VIC, Australia, ${ }^{5}$ Department of Biomedical Engineering, The University of Melbourne, Parkville, VIC, Australia

Several studies have illustrated that transcutaneous vagus nerve stimulation (tVNS) can elicit therapeutic effects that are similar to those produced by its invasive counterpart, vagus nerve stimulation (VNS). VNS is an FDA-approved therapy for the treatment of both depression and epilepsy, but it is limited to the management of more severe, intervention-resistant cases as a second or third-line treatment option due to perioperative risks involved with device implantation. In contrast, tVNS is a non-invasive technique that involves the application of electrical currents through surface electrodes at select locations, most commonly targeting the auricular branch of the vagus nerve (ABVN) and the cervical branch of the vagus nerve in the neck. Although it has been shown that tVNS elicits hypo- and hyperactivation in various regions of the brain associated with anxiety and mood regulation, the mechanism of action and influence of stimulation parameters on clinical outcomes remains predominantly hypothetical. Suppositions are largely based on correlations between the neurobiology of the vagus nerve and its effects on neural activity. However, tVNS has also been investigated for several other disorders, including tinnitus, migraine and pain, by targeting the vagus nerve at sites in both the ear and the neck. As most of the described methods differ in the parameters and protocols applied, there is currently no firm evidence on the optimal location for tVNS or the stimulation parameters that provide the greatest therapeutic effects for a specific condition. This review presents the current status of tVNS with a focus on stimulation parameters, stimulation sites, and available devices. For tVNS to reach its full potential as a non-invasive and clinically relevant therapy, it is imperative that systematic studies be undertaken to reveal the mechanism of action and optimal stimulation modalities.

Keywords: vagus nerve, vagus nerve stimulation, transcutaneous, neuromodulation, neurostimulation

\section{INTRODUCTION}

Vagus nerve stimulation (VNS) is an FDA-approved treatment for both pharmacoresistant depression and epilepsy and can produce clinically meaningful antidepressant and anti-seizure effects (Nemeroff et al., 2006; Johnson and Wilson, 2018). More than 100,000 VNS devices had been implanted in more than 70,000 patients globally by 2013 (Labiner and Ahern, 2007). The 
implantable device consists of an electrode, which is wrapped around the left vagus nerve, and an implantable unit, positioned below the collarbone and containing the battery and pulse generator.

Device implantation is predominantly performed on an outpatient basis under general anesthetic, but some patients may require overnight stay if extended observation is necessary. Despite being a minimally invasive procedure, the surgery is inherently risky due to the location of implantation, with electrode placement requiring dissection of the vagus nerve from the carotid artery. Potential adverse events arising from the surgical intervention include bradyarrhythmias during device placement, the development of peritracheal hematoma (due to surgical trauma), and other respiratory complications, including vocal cord dysfunction and dyspnea (due to nerve trauma). VNS can also cause changes to breathing patterns during sleep, resulting in an increase in the number of obstructive apneas and hypopneas (Marzec et al., 2003; Fahy, 2010), and can, albeit rarely, produce late-onset bradyarrhythmias and severe asystolia due to atrium-ventricular block (Iriarte et al., 2009). These potential adverse events limit the intervention's applicability to those who are resistant to conventional therapeutic strategies, and total device and procedural costs amount to around AU $\$ 50,000$ (Lehtimäki et al., 2013), a price that is prohibitively high for many, as it is a non-subsidized treatment.

Transcutaneous vagus nerve stimulation (tVNS) is a method that has been developed to overcome these limitations, and the potential widespread accessibility of the technology adds to its appeal as a possible first-line treatment option. Anatomical studies of the ear suggest that the tragus, concha, and cymba concha are the places on the human body where there are cutaneous afferent vagus nerve distributions (Figure 1) (Peuker and Filler, 2002), and it is believed that stimulation of these afferent fibers should produce therapeutic effects that are similar to those of regular VNS (Hein et al., 2012; Rong et al., 2012; Stefan et al., 2012). Similarly non-invasive stimulation of the cervical branch of the vagus nerve has received popularity due to minimal side effects, low cost, and morbidity associated with the technique (Goadsby et al., 2014; Grazzi et al., 2014; Kinfe et al., 2015b). In this review, we refer to both auricular and cervical nerve stimulation as tVNS.

The potential of tVNS is not limited to the treatment of depression and epilepsy, with the technology being investigated for a variety of disorders including headache, tinnitus, atrial fibrillation, post-error slowing, prosocial behavior, associative memory, schizophrenia, and pain (Laqua et al., 2014; Hasan et al., 2015; Hyvärinen et al., 2015; Jacobs et al., 2015; Nesbitt et al., 2015; Sellaro et al., 2015a,b; Stavrakis et al., 2015).

Despite the breadth of research being undertaken, many questions remain regarding the most effective stimulation sites and parameters. As many of the described methods differ in the parameters and protocols applied, there is currently no firm evidence regarding the optimal location for stimulation to achieve the greatest clinical effects let alone an understanding of the neurophysiological mechanisms. Therefore, this critical review aims to explore the reported studies in tVNS with a view to promoting more systematic approaches that might help to translate the technique into mainstream clinical practice.

In comparison to tVNS, the invasive approach to VNS has been the subject of a number of recent reviews. For example, a review of functional neuroimaging studies in VNS confirmed that invasive stimulation causes changes in various brain regions and at different levels (Chae et al., 2003). A review of VNS with a focus on depression is presented in Müller et al. (2018). Recent advances in devices for VNS have been covered in Mertens et al. (2018). Similarly, applications and potential mechanisms of VNS have been discussed in some detail (Groves and Brown, 2005; Yuan and Silberstein, 2016a,b).

The few reviews that specifically focus on tVNS are very recent. A systematic review of the safety and tolerability of tVNS was presented in Redgrave et al. (2018), while two companion papers have focused on the physiological and engineering perspectives of tVNS (Kaniusas et al., 2019a,b). Whereas, Kaniusas et al. (2019a,b) outlined current research directions in auricular vagus nerve stimulation, this review takes a more critical approach and explores fundamental limitations of study design protocols that may lead to difficulties in translating current research into the clinic. We have also reviewed cervical vagus nerve stimulation in addition to auricular applications.

The review presented here focuses on a mechanistic understanding of $\mathrm{tVNS}$, with a detailed description of stimulation parameters, sites of stimulation, and devices used in current research. We review current publications investigating the effect of electrode placement on auricular vagus nerve stimulation recruitment and corresponding neural activations, papers studying the effect of stimulation parameters (waveform, polarity, frequency, pulse width, duty cycle, and current), and manuscripts exploring the neurophysiological mechanisms of tVNS. We also consider whether tVNS can be used for closed-loop control of neural activity. We outline fundamental gaps in our understanding that need to be overcome in order to maximize efficacy, minimize risk, and thus support the successful translation of tVNS into mainstream clinical practice.

\section{TRANSCUTANEOUS VAGUS NERVE STIMULATION (tVNS)}

\subsection{Anatomical Considerations}

Transcutaneous vagus nerve stimulation (tVNS) is based on the results of anatomical studies illustrating the path of the auricular branch of the vagus nerve (ABVN; Alderman's nerve; and Arnold's nerve), which originates from the superior ganglion of the vagus nerve from within the jugular foramen (Tekdemir et al., 1998), transversely passing through the facial canal, entering the small canal of the petrous bone, and emerging from the tympanomastoid fissure, proceeding to innervate the external acoustic meatus and auricle (Kiyokawa et al., 2014). As Peuker and Filler identify, the ABVN (Figure 2) is most prominently spread through the antihelix, tragus, cymba concha, and concha (Peuker and Filler, 2002). These are the places on the human body where there are cutaneous afferent vagus nerve distributions, and thus, as theoretically proposed by Ventureyra 

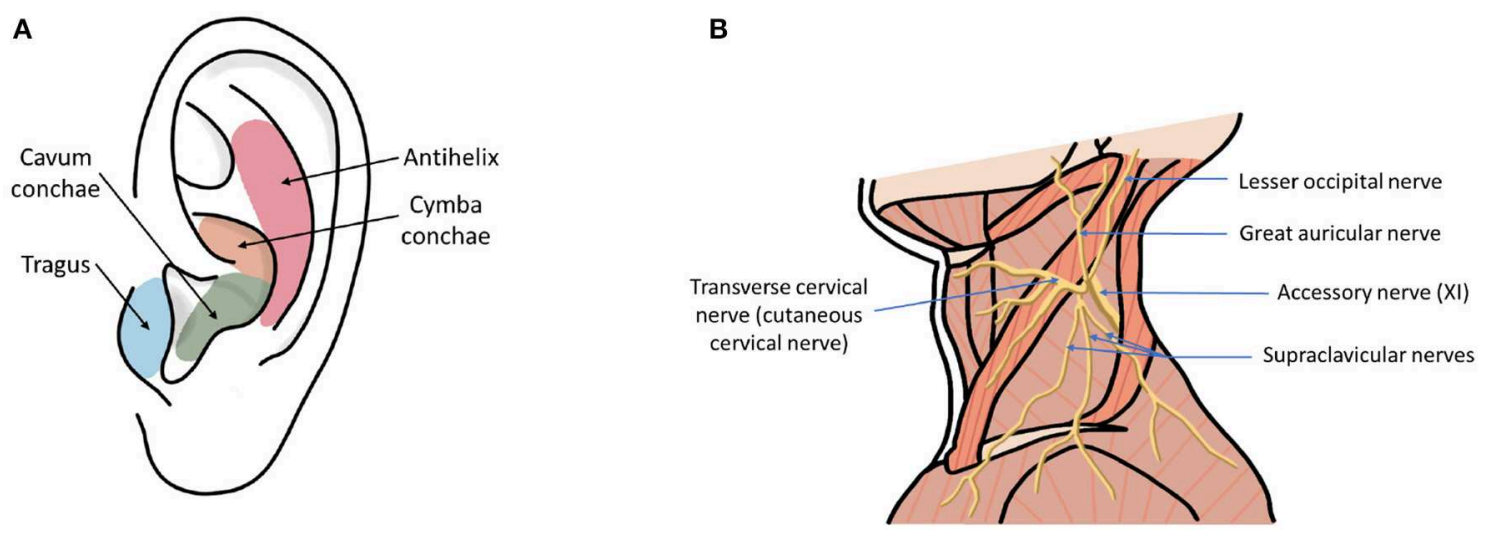

FIGURE 1 | (A) Ear regions with innervation by the cutaneous auricular branch of the vagus nerve (ABVN). (B) Nerves in the neck region including cervical branch of the vagus nerve.

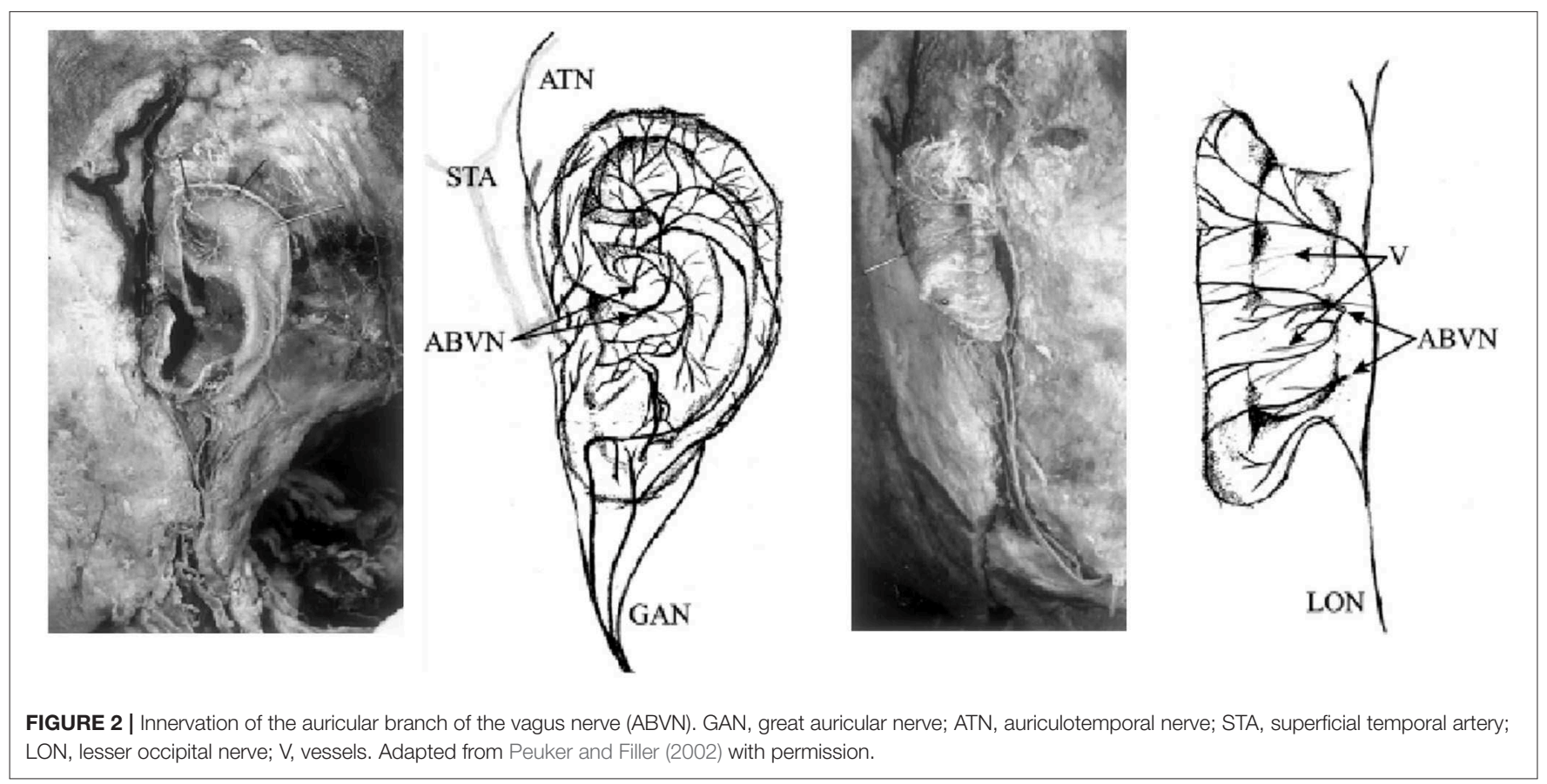

(2000), it is believed that direct stimulation of these nerve fibers should produce therapeutic effects similar to those of VNS. More recently, the original article by Peuker and Filler was the subject of some controversy due to different numbers being reported for tragus innervation by the ABVN in the main text and in the table (possibly a typing error) (Burger and Verkuil, 2018). Peuker and Filler (2002) later explained that the knowledge of auricular vagus nerve anatomy does not rest solely on this data, and other publications support the same findings (He et al., 2012).

Transcutaneous cervical vagus nerve stimulation is another method that has been developed to non-invasively stimulate the vagus nerve with electrodes placed over the sternocleidomastoid muscle. This is a similar location to where the electrodes for VNS are positioned and is more reminiscent of Corning's initial approach. However, the vagus nerve's location within the carotid sheath (Figure 3), beneath the skin $(2 \mathrm{~mm})$, superficial fascia (3-6 mm), and sternocleidomastoid muscle (5-6 mm) (Seiden et al., 2013) can make selective transcutaneous stimulation of vagus nerve fibers difficult, with current product offerings most likely indiscriminately stimulating afferent and efferent fibers alike (Yuan and Silberstein, 2016b).

Conventionally, the left vagus nerve has mostly been selected as the preferred stimulation site due to safety concerns arising from observations during animal studies showing that rightsided VNS results in a greater degree of bradycardia (Yuan and Silberstein, 2016b). This is due to the asymmetric innervation of the heart, where the right vagus nerve predominantly innervates the sinoatrial (SA) node and the left predominantly innervates 


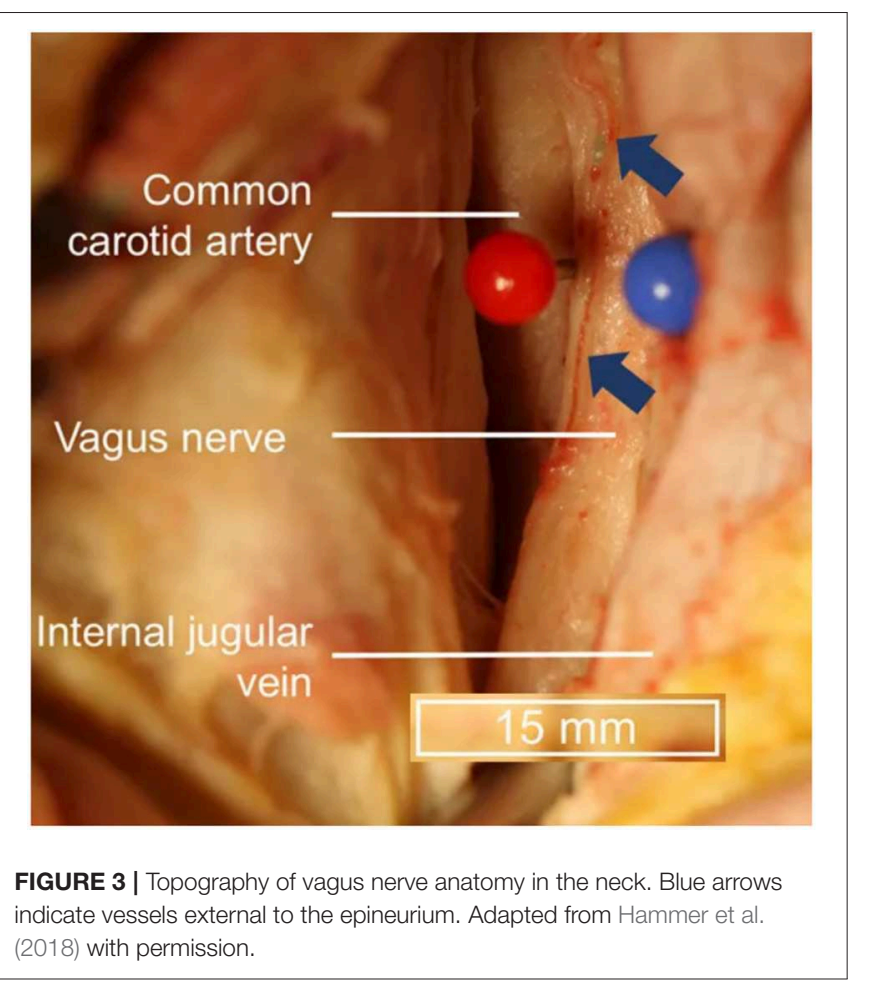

the atrioventricular (AV) node (Ardell and Randall, 1986). As such, right VNS in dog studies activated the cardiac motor efferents innervating the SA node, causing bradycardia through a reduction of depolarization rates and providing credence to the belief that right-sided VNS should not be attempted in clinical settings (Krahl, 2012). However, the anatomy of the cervical vagus trunk differs between dogs and humans, and the location around which the VNS stimulation electrodes are wrapped (in humans) does not include the superior or inferior cardiac branches, thereby diminishing the risk of significant cardiac adverse events (Krahl, 2012). Despite this, the FDA-approved labeling for VNS devices specifies that "the VNS Therapy System is indicated for use only in stimulating the left vagus nerve in the neck area inside the carotid sheath. The VNS Therapy System is indicated for use only in stimulating the left vagus nerve below where the superior and inferior cervical cardiac branches separate from the vagus nerve. The safety and efficacy of the VNS Therapy System have not been established for stimulation of the right vagus nerve or of any other nerve, muscle, or tissue" (Depression Physician's Manual, 2005).

While limiting treatments to the left side may be warranted for VNS, due to the potential to directly stimulate the cardiac motor efferents innervating the SA node, there are questions as to whether the application of these conventional reservations to tVNS is justified. The cardiac effects seen through ABVN stimulation are mediated through a neural pathway that involves the nucleus tractus solitarii (NTS); this activates the dorsal motor nucleus, which then delivers processed signals to the heart surface bilaterally via the efferent cervical vagus nerves. Therefore, unlike cervical VNS, tVNS circumvents the risk of
TABLE 1 | Classification of nerve fibers.

\begin{tabular}{|c|c|c|c|c|c|}
\hline Nerve fiber & Diameter & Myelination & $\begin{array}{l}\text { Conductic } \\
\text { velocity }\end{array}$ & Afferent or & Type \\
\hline classification & $(\mu \mathrm{m})$ & & $(\mathrm{m} / \mathrm{s})$ & Efferent & \\
\hline $\mathrm{A} \alpha$ & $13-20$ & Thick & 80-120 & Both & $\begin{array}{l}\text { Sensory } \\
\text { and Motor }\end{array}$ \\
\hline $\mathrm{A} \beta$ & 6-12 & Medium & 33-75 & Both & $\begin{array}{l}\text { Sensory } \\
\text { and Motor }\end{array}$ \\
\hline$A \gamma$ & $5-8$ & Medium & $4-24$ & Efferent & Motor \\
\hline $\mathrm{A} \delta$ & $1-5$ & Thin & $3-30$ & Afferent & Sensory \\
\hline B & $<3$ & Thin & $3-14$ & Afferent & Autonomic \\
\hline C & $0.2-1.5$ & None & $0.5-2$ & Afferent & $\begin{array}{l}\text { Sensory } \\
\text { and Motor }\end{array}$ \\
\hline
\end{tabular}

Adapted from Fix and Brueckner (2009).

directly and asymmetrically stimulating cardiac motor efferent fibers, thus causing adverse cardiac events (Chen et al., 2015). As such, simply disregarding the therapeutic potential of bilateral ABVN stimulation, based on conventional preconceptions and parallels drawn from VNS, may be premature and warrants further investigation. Additionally, bilateral ABVN stimulation has been shown to be safe in pilot studies investigating tVNS as a complementary therapy for pediatric epilepsy (He et al., 2013).

\subsection{Nerve Fiber Types}

The vagus and its branches consist of around 80\% sensory afferent and 20\% motor afferent fibers (Yu et al., 2008). Nerve fibers can be further classified into one of three groups based on their diameter: the A group (consisting of $\mathrm{A} \alpha \mathrm{A} \beta, \mathrm{A} \gamma$, and $\mathrm{A} \delta$ ), $\mathrm{B}$ group, and $\mathrm{C}$ group. The different nerve fiber types have different diameters and myelination thicknesses (Table 1), which corresponds to different conduction velocities, with thicker myelination typically linked to faster conduction velocities or signal propagation (Fix and Brueckner, 2009).

A-group fibers are thick, myelinated, afferent, and efferent, and they also typically have diameters of around 1-22 $\mu \mathrm{m}$ and a conduction velocity of $5-120 \mathrm{~m} / \mathrm{s}$. They are typically found in both motor and sensory pathways. B fibers are only moderately myelinated, with diameters $=3 \mu \mathrm{m}$ and a conduction velocity ranging from 3 to $15 \mathrm{~m} / \mathrm{s}$. C fibers are non-myelinated, and they thus have slower conduction speeds of $2 \mathrm{~m} / \mathrm{s}$ and thinner diameters of between 0.2 and $1.5 \mu \mathrm{m}$.

The cervical branch vagus nerve is made up of about $20 \%$ myelinated $\mathrm{A}$ and $\mathrm{B}$ fibers and $80 \%$ unmyelinated $\mathrm{C}$ fibers (Vonck et al., 2009). Contrary to earlier studies, which have suggested that $\mathrm{C}$ fiber recruitment during VNS was essential for seizure suppression, Kraus et al. (2007) showed that destruction of peripheral C fibers did not influence VNS-induced seizure suppression, and the therapeutic effects of VNS have thus been attributed to the maximal recruitment of thick afferent A and B nerve fibers (Evans et al., 2004). Minimal side effects suggest that stimulation of these fibers is well-tolerated (Helmers et al., 2012).

Similarly, Stefan et al. (2012) showed that tVNS does not elicit painful sensations in the participants, which suggests that afferent $\mathrm{C}$ axons and thin myelinated $\mathrm{A} \delta$ axons are not activated. 
A study by Mourdoukoutas et al. (2018) also investigated the fibers that can be activated by tVNS, and they found that at the typically used current of $10 \mathrm{~mA}$, only A-axons and larger B-axons were activated; this is likely due to the diameter of their fibers, implying that C-fibers were too thin to be activated by the applied electrical stimulation.

At the cervical level, the vagus nerve mainly consists of small diameter unmyelinated $C$ fibers (65-80\%) and of a smaller portion of intermediate- diameter myelinated $\mathrm{B}$ fibers and largediameter myelinated A fibers. A, B, and C fiber distributions within the carotid vagus nerve have been well-documented (Standring, 2015), enabling the development of computational models to determine the optimal current and pulse width parameters for VNS to activate the myelinated A and B afferent fibers (Helmers et al., 2012). Despite this, the optimal stimulation parameters for VNS are unknown, as the effects of other parameters, such as frequency and duty cycle, are observed post-synaptically in various structures of the brain. Given that these activations cannot be computationally modeled, clinical application and stimulation parameter selection of VNS relies on subjective benefits reported by patients.

In contrast, the distributions of the various nerve fiber types of the ABVN have not been investigated to the level of detail necessary for computational modeling. Therefore, the presence of various nerve fiber types remains speculative and evaluations of intervention efficacy have been based on subjectively experienced therapeutic benefits correlated with other primary and secondary outcomes, such as neuroimaging studies.

As with stimulation of the cervical branches of the vagus nerve with low level electrical currents, stimulation of the ABVN would be expected to activate thick myelinated fibers only and with no activation of the thin diameter unmyelinated $C$ fibers. The $\mathrm{ABVN}$ is a general sensory fiber and is one of the few branches to contain no motor fibers. As such, the myelinated fibers found in the ABVN would be expected to be A-group sensory axons rather than B-group autonomic fibers. Only one study has determined the number of myelinated axons that are present in the ABVN (Safi et al., 2016). Around $50 \%$ of the myelinated axons were measured to have a diameter of between 2.5 and $4.4 \mu \mathrm{m}$, which suggests that they belong to the A $\delta$ group. Nearly $20 \%$ of the axons were measured to have a diameter $>7 \mu \mathrm{m}$, suggesting the fibers belong to the $\mathrm{A} \beta$ class. However, the ABVN contains almost six times less $\mathrm{A} \beta$ class nerve fibers than those found in the cervical branch of the vagus nerve. This number also varied greatly between individuals, which may explain why some individuals do not experience therapeutic effects after treatment with tVNS, and it may go some way to explain the anatomical basis behind the mechanism and effectiveness of tVNS (Butt et al., 2019).

\section{3. tVNS for Common Health Conditions}

\subsubsection{Depression}

The mechanism behind the therapeutic anti-depressive effects of VNS and tVNS is still unknown. In 2007, Kraus et al. investigated the acute brain activations of healthy subjects following tVNS through functional magnetic resonance imaging (fMRI), showing hypoactivation of the amygdala, hippocampus, parahippocampal gyrus, and middle and superior temporal gyrus, and hyperactivation in the insula, precentral gyrus, and thalamus (Kraus et al., 2007). These cortical areas are connected both directly and indirectly to the nucleus tractus solitarii (NTS), which receives greatest afferent vagus input. The NTS relays incoming sensory information to the brain via an automatic feedback loop, direct projections to the reticular formation in the medulla, and ascending projections to the amygdala, insula, hypothalamus, thalamus, orbitofrontal cortex, and other limbic regions involved in anxiety and mood regulation via the parabrachial nucleus and the locus coeruleus (Mohr et al., 2011). It is hypothesized that hypoactivation of the amygdala suppresses the hyperactive limbic brain areas, as seen in patients with depression (Mayberg, 1997), through projections from the amygdala to the amygdala-hippocampus-entorhinal cortex of the limbic system (Kraus et al., 2007).

These results are consistent with the acute diminished activity of the limbic system found during VNS (Henry et al., 1998; Chae et al., 2003; Mohr et al., 2011). Interestingly, changes in regional cerebral blood flow induced by VNS are similar to those found in depressed patients treated with selective serotonin reuptake inhibitors (fluoxetine) (Mayberg et al., 2000), either in the amygdala, hippocampus, or parahippocampus (Nemeroff et al., 2006). fMRI studies of patients with depression, following 1 month of tVNS, showed increased functional connections between the default mode network and the precuneus, rostral anterior cingulate cortex, and medial prefrontal cortex. This has also been associated with a reduction in depression severity (Fang et al., 2016) and is similar to results illustrating the therapeutic effects of transcranial magnetic stimulation (Fitzgerald et al., 2006).

Activation of the central nervous system via electrical stimulation of peripheral nerves has become known as the "bottom-up" mechanism, which is a hypothesis based on the neurobiology of the vagus nerve and its effects on neural activity. This is in contrast to the well-known "top-down" mechanism of strategies, such as electroconvulsive therapy and transcranial magnetic stimulation, where the stimulus is applied to central brain structures and subsequently propagates to peripheral sites (Shiozawa et al., 2014). In both human and animal studies, VNS has been shown to elicit changes in neurotransmitters associated with the pathophysiology of depression, including serotonin, norepinephrine, GABA, and glutamate (Ben-Menachem et al., 1995; Krahl et al., 1998; Walker et al., 1999; Dorr and Debonnel, 2006; Manta et al., 2009).

Hein et al. (2012) illustrated the antidepressant effects of 2 weeks of tVNS using an add-on study design, which resulted in significantly improved outcomes on the Beck Depression Inventory (BDI; 27.0-14.0 points). However, no significant changes were observed on the Hamilton Depression Rating Scale (HAMD). Very little information was provided regarding the stimulation parameters that were used; $1.5 \mathrm{~Hz}$ unipolar rectangular waves and currents were individually adjusted to maximal but not painful intensities (0-600 mA). In a single blinded clinical trial conducted by Fang et al. (2016) investigating the antidepressant effects of tVNS as a solo treatment, significant improvement was not only seen on the HAMD (28.5-15.0) but 
also on the Self-Rating Anxiety Scale (SAS; 56.56-42.83) and the Self-Rating Depression Scale (SDS; 66.33-50.56). It is implied that these therapeutic effects may be due to modulation of the resting state functional connectivity of the default mode network, as shown via fMRI imaging. Again, the stimulation parameters used were not comprehensively reported, with density wave adjusted to $20 \mathrm{~Hz}$, a wave width $<1 \mathrm{~ms}$, and intensity adjusted based on the tolerance of the patient (4-6 mA).

\subsubsection{Epilepsy}

In addition to depression, tVNS has also been investigated for its use as a treatment option for drug-resistant epilepsy, a neurological disorder characterized by recurring seizures that affects around 50 million people worldwide (Beghi, 2019). Drug resistance is diagnosed in up to $30 \%$ of epilepsy patients (Kwan and Brodie, 2000). Handforth et al. (1998) demonstrated that invasive stimulation of the vagus nerve could suppress the occurrence of seizures and offer a non-pharmacological treatment for epilepsy.

Due to the success of invasive vagus nerve stimulation as a valid treatment option for epilepsy, Stefan et al. (2012) devised a pilot study to investigate whether tVNS would elicit the same anti-convulsive effects. In the pilot study, 10 participants with drug-resistant epilepsy who experienced a minimum of four seizures a month were stimulated on the auricular branch of the vagus nerve transcutaneously through the tragus of the left ear. The stimulation parameters were set to a frequency of $10 \mathrm{~Hz}$ with a pulse width of $0.3 \mathrm{~ms}$, and the stimulation intensity was set to the individual's tolerance threshold. The participants were trained to self-administer the tVNS for three 1-h sessions per day as part of their daily routine over a period of 9 months. The participants were encouraged to keep a seizure diary to report the frequency of their seizures both before and during tVNS treatment. In five out of the seven cases that completed the study, the seizure frequency was reduced, which suggested that tVNS could offer seizurereduction effects.

He et al. (2013) also conducted a pilot study to investigate tVNS as a treatment option for pediatric epilepsy. The stimulation protocol differed to the study of Stefan et al. above, as the stimulation was delivered to the left concha with a frequency of $20 \mathrm{~Hz}$ for only $30 \mathrm{~min}$ at a time three times daily for 6 months. These parameters were found to also elicit seizure-reduction effects, with a $54 \%$ reduction in seizure frequency reported after the 6 months of tVNS treatment. More recently, Liu et al. (2018) found an average seizure reduction of $64.4 \%$ in 16 out of 17 of their patients after 6 months of treatment with tVNS. The participants were trained to administer $20 \mathrm{~min}$ of tVNS three times a day for 6 months to the left concha with a stimulation frequency of $10 \mathrm{~Hz}$.

The exact mechanism by which tVNS prevents or inhibits seizures is not well-understood. It is thought that afferent projections from the ABVN to the nucleus tractus solitarius (NTS) may be responsible for the anti-convulsive effect, however, the neural networks projecting downstream are unclear (Henry, 2002).

\subsubsection{Tinnitus}

Tinnitus is the perception of sound in the absence of actual external sound and it affects $10-15 \%$ of the general population (Han et al., 2009). Recent imaging studies have suggested that chronic tinnitus is linked to a dysfunction in the auditory system, which results in abnormal neuronal behavior. Pairing of invasive vagus nerve stimulation with sound therapy has been shown to reverse tinnitus in rat models (Engineer et al., 2011), and so Lehtimäki et al. (2013) devised a pilot study to investigate whether tVNS could provide any therapeutic benefits for patients with chronic tinnitus. In addition, they also investigated whether tVNS could affect neuronal activity in the auditory cortex by imaging the brain using magnetoencephalography (MEG).

During the study, 10 participants with chronic tinnitus were stimulated continuously on the left tragus at $25 \mathrm{~Hz}$ for $45-60$ min over seven sessions. The stimulation was paired with tailored sound therapy, which was classical music with the dominant frequency of the individual's tinnitus removed. After the study, all participants reported improved mood and decreased severity of tinnitus. In addition, MEG scans demonstrated that tVNS modulated the auditory cortical response, which suggests that the auditory system can be accessed and modulated via stimulation of the vagus nerve.

\subsubsection{Migraine}

A number of studies have looked at applying non-invasive VNS to the neck to treat migraines (Goadsby et al., 2014; Grazzi et al., 2014, 2016; Barbanti et al., 2015; Kinfe et al., 2015b). In all of these studies, the gammaCore device (ElectroCore, 2018) was held against the neck in the region of the cervical branch of the vagus nerve, where two stainless steel electrodes deliver 25 $\mathrm{Hz}$ of burst stimulation. Total stimulation time varies between studies, but most give $90 \mathrm{~s}$ doses of stimulation at a time. This approach has found success in not only reducing the frequency of migraine attacks in participants but also the severity and resultant disability of the attacks.

In addition to non-invasive VNS at the neck, Straube et al. (2015) also investigated whether tVNS at the tragus would have a similar therapeutic effect on migraine. They devised a study for 46 participants, testing the NEMOS tVNS device applying $25 \mathrm{~Hz}$ to the tragus for $4 \mathrm{~h}$ per day over 3 months, and they also used $1 \mathrm{~Hz}$ to the tragus as an active control. Interestingly, the $1 \mathrm{~Hz}$ stimulation elicited a more significant reduction in the number of headache days than the $25 \mathrm{~Hz}$ active stimulation. This was an unexpected result and demonstrates that a more robust investigation into different stimulation parameters is crucial.

Again, the mechanism of non-invasive VNS and its effect on migraine is not well-understood. One possibility for the therapeutic effects of non-invasive vagus nerve stimulation is thought to be due to activation of the thalamus, which is responsible for information processing and regulation of cortical activity. In patients with migraine, fMRI studies have shown that there is a decrease in thalamocortical activity, and so stimulation of the vagus may help to counteract this decline (Coppola et al., 2004). Alternatively, it is possible that stimulation of the vagus nerve inhibits nociceptive trigeminal neurons, which may have a pain-inhibitory effect (Randich and Gebhart, 1992). 


\subsubsection{Pain}

Johnson et al. first attempted to study the effect of transcutaneous electrical stimulation of the ear on pain threshold in 1991, with a pilot study of 18 participants receiving low frequency burst stimulation at $2.3 \mathrm{~Hz}$ for $15 \mathrm{~min}$ on three different auricular sites (Johnson et al., 1991). In this study, pain threshold was noted to increase in 10 out of the 18 participants. Three participants also experienced a prolonged analgesic effect even after the stimulation device was turned off.

This pain-inhibitory effect was also noted by Multon and Schoenen (2005) in a review of clinical data collected from patients with implanted VNS devices. The pain thresholds of the patients and any effect VNS had on headaches was measured and confirmed that implanted VNS offered an analgesic effect. Following on from this review of implanted VNS devices, Laqua et al. (2014) proposed a study to investigate whether non-invasive tVNS could offer the same analgesic effect. Electrical stimulation was delivered for $30 \mathrm{~min}$ transcutaneously at the cavum conchae in burst stimulation mode with a changing frequency between 2 and $100 \mathrm{~Hz}$. The individual pain threshold was measured using a Neurometer device that measures the sensory nerve conduction threshold. Of the 21 participants, 15 responded with an increase in pain threshold during tVNS, while six noted a decrease in pain threshold during stimulation. These results, although contradictory, agree with the findings of Johnston et al. and support the view that the analgesic effects of VNS are very much dependent on individual sensitivity alongside stimulation parameters.

Busch et al. (2013) devised a study to investigate whether tVNS has the potential to alter pain processing by examining different submodalities of the somatosensory system. A total of 48 participants were stimulated at the left concha on the inner side of the tragus with a stimulation frequency of $25 \mathrm{~Hz}$. Different tests were devised to measure different pain thresholds, such as heat, mechanical, and pressure-related pain thresholds. The results showed an inhibition of mechanical, heat and pressure pain sensitivity after $1 \mathrm{~h}$ of continuous tVNS. Detection thresholds for thermal or mechanical inputs were not altered. These results suggest that tVNS can influence pain processing and offer an inhibitory effect on different pain modalities. Analysis of these different submodalities also suggests that tVNS has an impact on the central pain processing centers rather than just peripheral nociceptor activity.

\section{LIMITATIONS OF CURRENT STUDY PROTOCOLS}

While the use of tVNS has been shown to elicit therapeutic benefits through various studies (Hein et al., 2012; Lehtimäki et al., 2013; Mei et al., 2014; Straube et al., 2015; Liu et al., 2018), they mostly use different primary and secondary outcome measures and so the comparability between studies is limited. While this is partly due to the application of the technique to various ailments where primary efficacy endpoints differ between studies, there are also major issues with incomplete reporting and inconsistent use of terminology when reporting the results of incomparable and, in some cases, non-reproducible experiments. The stimulation parameters, devices, electrode types and the main findings of relevant studies are summarized in Table 2.

\subsection{Stimulation Devices}

Research groups generally report the stimulation device used in the experiment, but many of the models used have now been discontinued, and access to their technical specifications is limited. The most commonly used devices are the gammaCore electroCore or Nemos Cerbomed (Figure 4), with a third of the studies included in Table 2 employing them for stimulation (e.g., Grazzi et al., 2014, 2016; Frangos et al., 2015; Straube et al., 2015; Frokaer et al., 2016; Lerman et al., 2016; Silberstein et al., 2016a,b). Almost always, the gammaCore electroCore device is used for stimulation at a neck site (e.g., Goadsby et al., 2014; Grazzi et al., 2014, 2016; Lerman et al., 2016; Silberstein et al., 2016a,b) whilst the NEMOS Cerbomed device is predominantly used for stimulation of the ABVN in the ear. The next most common stimulation device is CM02 Cerbomed, used in Sellaro et al. (2015a,b), Hasan et al. (2015), and Steenbergen et al. (2015) among others. The gammaCore or NEMOS devices are often selected for convenience as they provide an easy-to-use package that includes stimulation electrodes. On the other hand, devices, such as TENS-200 or Digitimer DS7A often require custommade electrodes. The NMS 300 device from Xavant Technology has also been used (Schulz-Stübner and Kehl, 2011), while the device has not been specified in two studies (Gaul et al., 2016).

\subsubsection{ElectroCore Gammacore}

The gammaCore, marketed by electroCore, is a handheld tVNS device that stimulates the vagus nerve within the cervical carotid sheath. The device has been granted investigational FDA approval for the acute and/or prophylactic treatment of primary headache and medication overuse headache in adults. Conductive gel is applied to the stimulation surfaces, which are then placed over the sternocleidomastoid muscle. Stimulation intensity is user-controlled (up to $24 \mathrm{~V}$ and $60 \mathrm{~mA}$ ), with individual treatment sessions lasting for $120 \mathrm{~s}$. The treatment can be safely administered multiple times per day; having been applied up to 6-12 times per day in clinical studies (Yuan and Silberstein, 2016b). The remaining stimulation parameters are fixed, delivering $1 \mathrm{~ms}$ pulses of $5 \mathrm{kHz}$ sine waves at 25 Hz. It delivers a proprietary pulse waveform that is designed to penetrate through various levels of tissue, including skin, muscle, and nerve sheaths, in order to stimulate the afferent vagus nerve fibers within the carotid sheath. Potential side effects can include tingling under the stimulation electrodes and mild facial twitching at high intensities. It is a limited-use device that is available in two models: 50 doses and 150 doses. Optimal device usage, in terms of the number of stimulations per day and/or total stimulation duration, is yet to be determined.

\subsubsection{Cerbomed NEMOS}

The NEMOS device (distributed by tVNS Technologies, previously Cerbomed) is a portable transcutaneous electrical nerve stimulator that delivers stimulus to ABVN distributions located in the left cymba concha. NEMOS has been granted the 
TABLE 2 | Summary of previous tVNS clinical trials and studies.

References Condition/Study Participants tVNS device Electrode type

Electrode type Stimulation Stimulation

Sham

Pulse width

Intensity

Freq $(\mathrm{Hz})$

Duty

cycle/Time

Brain activation

\begin{tabular}{llllllll}
\hline Keute et al. & Visual bistable & 34 & Digitimer DS7 & $\mathrm{Ag} / \mathrm{AgCl}$ & $\mathrm{L}$ & Cymba Concha & Shan
\end{tabular}

Sham $0.2 \mathrm{~ms}$

$3 \mathrm{~mA}$

$25 \mathrm{~Hz}$

$30 \mathrm{~s}$ on, $30 \mathrm{~s}$ stimulation 25

$\mathrm{Hz}$ on

$30 \mathrm{~s}$ on, $30 \mathrm{~s}$
off for $40 \mathrm{~min}$

Inferred-tVNS has nu effect on dynamics of visual bistable perception

列

motor bu

$\begin{array}{llll}\text { Zhao et al. } & \text { Post-stroke } & 1 & \text { NS }\end{array}$

L, R Concha

NS

$<1 \mathrm{~ms}$

$4-6 \mathrm{~mA}$

$20 \mathrm{~Hz}$

30 min twice a

day for 4

Measured-Bold fMRI

showed a decrease in

functional connectivity

between posterior cingulate

cortex and other nodes of

default mode network but a

decrease in functiona

connectivity between

posterior cingulate cortex

lingual gyrus, and cortex

surrounding calcarine

$\begin{array}{lllll}\text { Badran et al. Improving } & 5 & \text { Digitimer } & \text { Custom ear electrode } L & \text { Tragus }\end{array}$

Tagus

NS

$0.5 \mathrm{~ms}$

$0.1 \mathrm{~mA}$
below

perception in newborns

DSTAH

threshold

$25 \mathrm{~Hz}$

Max 2 min or

less per dose,

paired with

newborn

feeding, stops

when newborn

stops sucking,

up to $30 \mathrm{~min}$ a

day over

$\begin{array}{lllll}\begin{array}{l}\text { Badran et al. } \\ \text { (2018a) }\end{array} & \begin{array}{l}\text { Neuro-physiologic } \\ \text { effects of tVNS }\end{array} & 17 & \text { Digitimer DS7 } & \text { Ag/AgCl }\end{array}$

Tragus

Sham $\quad 0.5 \mathrm{~ms} \quad 200 \%$ of

stimulation $25 \quad$ perception

$25 \mathrm{~Hz} \quad 3 \times 60$ sover

Measured-Bold fMRI

$\mathrm{Hz}$ on ear

threshold

$6 \mathrm{~min}$

showed active stimulation

produced significantly

greater increases in the

right caudate, bilateral

anterior cingulate,

cerebellum, left prefrontal

cortex, and mid-cingulate

than in sham stimulation

\begin{tabular}{|c|c|c|c|c|c|c|c|c|c|c|c|c|}
\hline $\begin{array}{l}\text { Colzato et al. } \\
\text { (2018) }\end{array}$ & Divergent thinking & 80 & $\begin{array}{l}\text { NEMOS, } \\
\text { Cerbomed }\end{array}$ & Titanium* & L & Concha & $\begin{array}{l}\text { Sham } \\
\text { stimulation } 25 \\
\text { Hz on ear } \\
\text { lobe }\end{array}$ & $0.2-0.3 \mathrm{~ms}$ & $0.5 \mathrm{~mA}$ & $25 \mathrm{~Hz}$ & $\begin{array}{l}30 \mathrm{~s} \mathrm{on}, 30 \mathrm{~s} \\
\text { off for } 40 \mathrm{~min}\end{array}$ & $\begin{array}{l}\text { Inferred-tVNS enhances } \\
\text { creativity in selective ways, } \\
\text { increased divergent } \\
\text { thinking which may be } \\
\text { attributed to possible } \\
\text { increase in GABA } \\
\text { concentration }\end{array}$ \\
\hline $\begin{array}{l}\text { Fischer et al. } \\
\text { (2018) }\end{array}$ & $\begin{array}{l}\text { Conflict-triggered } \\
\text { adjustment of } \\
\text { cognitive control }\end{array}$ & 21 & $\begin{array}{l}\text { CM02, } \\
\text { Cerbomed }\end{array}$ & Two titan electrodes & L & Cymba Concha & $\begin{array}{l}\text { Sham } \\
\text { stimulation } \\
25 \mathrm{~Hz} \text { on ear } \\
\text { lobe }\end{array}$ & $0.2-0.3 \mathrm{~ms}$ & $\begin{array}{l}\text { Below pain } \\
\text { threshold } \\
\text { (average } \\
1.3 \mathrm{~mA} \text { ) }\end{array}$ & $25 \mathrm{~Hz}$ & $\begin{array}{l}\text { Continuously } \\
\text { for } 36 \text { min }\end{array}$ & $\begin{array}{l}\text { Measured-EEG showed } \\
\text { tVNS increasing behavioral } \\
\text { and electrophysiological } \\
\text { markers of conflict } \\
\text { adaptation }\end{array}$ \\
\hline
\end{tabular}


TABLE 2 | Continued

\begin{tabular}{|c|c|c|c|c|c|c|c|c|c|c|c|c|}
\hline References & Condition/Study & Participants & tVNS device & Electrode type & $\begin{array}{l}\text { Stimulation } \\
\text { Side }\end{array}$ & $\begin{array}{l}\text { Stimulation } \\
\text { Site }\end{array}$ & $\begin{array}{l}\text { Sham } \\
\text { control }\end{array}$ & $\begin{array}{l}\text { Pulse width } \\
\text { (ms) }\end{array}$ & $\begin{array}{l}\text { Intensity } \\
(\mathrm{mA})\end{array}$ & Freq $(\mathrm{Hz})$ & $\begin{array}{l}\text { Duty } \\
\text { cycle/Time }\end{array}$ & Brain activation \\
\hline $\begin{array}{l}\text { Jongkees et al. } \\
\text { (2018) }\end{array}$ & $\begin{array}{l}\text { Response } \\
\text { selection during } \\
\text { sequential action }\end{array}$ & 40 & $\begin{array}{l}\text { CM02, } \\
\text { Cerbomed }\end{array}$ & Two titan electrodes & L & Tragus & $\begin{array}{l}\text { Sham } \\
\text { stimulation } 25 \\
\text { Hz on ear } \\
\text { lobe }\end{array}$ & $0.2-0.3 \mathrm{~ms}$ & $0.5 \mathrm{~mA}$ & $25 \mathrm{~Hz}$ & $\begin{array}{l}30 \mathrm{~s} \mathrm{on,} 30 \mathrm{~s} \\
\text { off for } 45 \mathrm{~min}\end{array}$ & $\begin{array}{l}\text { Inferred-tVNS improves } \\
\text { response selection, } \\
\text { possibly due to tVNS } \\
\text { increasing GABA } \\
\text { concentration, which } \\
\text { facilitates action control }\end{array}$ \\
\hline $\begin{array}{l}\text { Keute et al. } \\
\text { (2018) }\end{array}$ & $\begin{array}{l}\text { GABAergic } \\
\text { modulation }\end{array}$ & 16 & Digitimer DS7 & Ambu Neuroline & L & Concha & $\begin{array}{l}\text { Sham } \\
\text { stimulation } 25 \\
\text { Hz on ear } \\
\text { lobe }\end{array}$ & $0.2 \mathrm{~ms}$ & $\begin{array}{l}8 \mathrm{~mA} \text { (or } \\
\text { below pain } \\
\text { threshold if } \\
\text { not } \\
\text { tolerable) }\end{array}$ & $25 \mathrm{~Hz}$ & $\begin{array}{l}30 \mathrm{~s} \text { on, } 30 \mathrm{~s} \\
\text { off for } 25 \mathrm{~min}\end{array}$ & $\begin{array}{l}\text { Measured-EEG } \\
\text { demonstrated direct } \\
\text { GABAergic effects of tVNS, } \\
\text { shows direct effect on } \\
\text { electrophysiology after } \\
\text { single session of tVNS and } \\
\text { suggests non-linear } \\
\text { relationship between tVNS } \\
\text { and GABA transmission }\end{array}$ \\
\hline Liu et al. (2018) & Epilepsy & 17 & $\begin{array}{l}\text { TENS-sm } \\
\text { device, Suzhou } \\
\text { Medical Audio } \\
\text { Supplies }\end{array}$ & Ear clip & $L, R$ & $\begin{array}{l}\text { Cymba Concha and } \\
\text { outer ear canal }\end{array}$ & NS & $200 \mathrm{~s}^{\dagger}$ & $\begin{array}{l}4 \mathrm{~mA} \\
\text { (increased } \\
\text { by } 2 \mathrm{~mA} \\
\text { each week } \\
\text { until } \\
\text { patient } \\
\text { could not } \\
\text { tolerate or } \\
\text { seizures } \\
\text { were } \\
\text { completely } \\
\text { controlled) }\end{array}$ & $10 \mathrm{~Hz}$ & $\begin{array}{l}3 \times 20 \text { min } \\
\text { daily for } 6 \\
\text { months }\end{array}$ & $\begin{array}{l}\text { Measured-tVNS reduced } \\
\text { the number of epileptic } \\
\text { seizures and reduced } \\
\text { abnormal wave changes } \\
\text { shown on } \\
\text { electroencephalogram } \\
\text { (EEG) monitoring. The EEG } \\
\text { changes followed the } \\
\text { reduction in the frequency } \\
\text { of seizures }\end{array}$ \\
\hline $\begin{array}{l}\text { Yakunina et al. } \\
\text { (2018) }\end{array}$ & Tinnitus & 36 & Custom-made & NS & L & $\begin{array}{l}\text { Inner tragus and } \\
\text { cymba concha }\end{array}$ & $\begin{array}{l}\text { Sham } \\
\text { stimulation } 25 \\
\text { Hz on ear } \\
\text { lobe }\end{array}$ & $0.5 \mathrm{~ms}$ & $\begin{array}{l}0.1 \mathrm{~mA} \\
\text { lower than } \\
\text { pain } \\
\text { threshold }\end{array}$ & $25 \mathrm{~Hz}$ & $\begin{array}{l}30 \mathrm{~s} \text { on, } 30 \mathrm{~s} \\
\text { off for } 6 \times 5 \\
\text { min runs }\end{array}$ & $\begin{array}{l}\text { Measured-fMRI showed } \\
\text { tVNS via both the tragus } \\
\text { and concha successfully } \\
\text { suppressed the auditory, } \\
\text { limbic, and other brain } \\
\text { areas inmlicated in the } \\
\text { mechanisms involved in the } \\
\text { generation/perception of } \\
\text { tinnitus via auditory and } \\
\text { vagal ascending pathways }\end{array}$ \\
\hline $\begin{array}{l}\text { Assenza et al. } \\
\text { (2017) }\end{array}$ & Epilepsy & 1 & $\begin{array}{l}\text { NEMOS, } \\
\text { Cerbomed }\end{array}$ & Titanium & L & $\begin{array}{l}\text { External acoustic } \\
\text { meatus }\end{array}$ & $\begin{array}{l}\text { Sham } \\
\text { stimulation on } \\
\text { right ear lobe }\end{array}$ & NS & $\begin{array}{l}\text { Sensitive } \\
\text { threshold }\end{array}$ & NS & $4 \mathrm{~h}$ & $\begin{array}{l}\text { Inferred: tVNS engages } \\
\text { same neural fibers as in } \\
\text { invasive VNS }\end{array}$ \\
\hline $\begin{array}{l}\text { Fang et al. } \\
(2017)\end{array}$ & Depression & 38 & $\begin{array}{l}\text { Suzhou Medical } \\
\text { Appliance } \\
\text { Factory }\end{array}$ & $\begin{array}{l}\text { Custom ear clip } \\
\text { electrodes }\end{array}$ & & Concha & $\begin{array}{l}\text { Sham } \\
\text { stimulation } 20 \\
\mathrm{~Hz} \text { delivered } \\
\text { to superior } \\
\text { scapha }\end{array}$ & $0.2 \mathrm{~ms}$ & $\begin{array}{l}\text { Tolerance } \\
\text { threshold } \\
\text { (typically } \\
\text { between } 4 \\
\text { and } 6 \mathrm{~mA} \text { ) }\end{array}$ & $20 \mathrm{~Hz}$ & $\begin{array}{l}\text { Continuously } \\
\text { for } 30 \text { min } \\
\text { twice a day, } 5 \\
\text { days a week } \\
\text { for } 4 \text { weeks }\end{array}$ & $\begin{array}{l}\text { Measured-fMRI shows } \\
\text { that tVNS targets left } \\
\text { anterior insula, and } \\
\text { activation of this region } \\
\text { predicts the outcome of } \\
\text { treatment for depression }\end{array}$ \\
\hline
\end{tabular}


TABLE 2 | Continued

\begin{tabular}{|c|c|c|c|c|c|c|c|c|c|c|c|c|}
\hline References & Condition/Study & Participants & tVNS device & Electrode type & $\begin{array}{l}\text { Stimulation } \\
\text { Side }\end{array}$ & $\begin{array}{l}\text { Stimulation } \\
\text { Site }\end{array}$ & $\begin{array}{l}\text { Sham } \\
\text { control }\end{array}$ & $\begin{array}{l}\text { Pulse width } \\
\text { (ms) }\end{array}$ & $\begin{array}{l}\text { Intensity } \\
(\mathrm{mA})\end{array}$ & Freq $(\mathrm{Hz})$ & $\begin{array}{l}\text { Duty } \\
\text { cycle/Time }\end{array}$ & Brain activation \\
\hline Yu et al. (2017) & $\begin{array}{l}\text { Disorders of } \\
\text { consciousness }\end{array}$ & 1 & NS & NS & L, R & Concha & NS & $<1 \mathrm{~ms}$ & $4-6 \mathrm{~mA}$ & $20 \mathrm{~Hz}$ & $\begin{array}{l}30 \text { min twice a } \\
\text { day for } 4 \\
\text { weeks }\end{array}$ & $\begin{array}{l}\text { Measured-fMRI shows } \\
\text { that tVNS activated } \\
\text { posterior } \\
\text { cingulate/precuneus and } \\
\text { thalamus and increased the } \\
\text { functional connectivity } \\
\text { between posterior } \\
\text { cingulate/precuneus and } \\
\text { hypothalamus, thalamus, } \\
\text { ventral medial prefrontal } \\
\text { cortex (vmPFC), superior } \\
\text { temporal gyrus, yet } \\
\text { decreased the functional } \\
\text { connectivity between } \\
\text { posterior } \\
\text { cingulate/precuneus and } \\
\text { the cerebellum }\end{array}$ \\
\hline $\begin{array}{l}\text { Bauer et al. } \\
\text { (2016) }\end{array}$ & Epilepsy & 76 & $\begin{array}{l}\text { NEMOS, } \\
\text { Cerbomed }\end{array}$ & Titanium ${ }^{*}$ & L & Cymba Concha & $\begin{array}{l}\text { Active control } \\
1 \mathrm{~Hz} \\
\text { stimulation }\end{array}$ & $0.25 \mathrm{~ms}$ & $\begin{array}{l}\text { Tingling } \\
\text { without } \\
\text { pain }\end{array}$ & 25 or $1 \mathrm{~Hz}$ & $\begin{array}{l}30 \mathrm{~s} \text { on } 30 \mathrm{~s} \\
\text { off for } 4 \mathrm{~h}\end{array}$ & NS \\
\hline $\begin{array}{l}\text { Burger et al. } \\
\text { (2016) }\end{array}$ & $\begin{array}{l}\text { Fear extinction in } \\
\text { health volunteers }\end{array}$ & 38 & $\begin{array}{l}\text { NEMOS, } \\
\text { Cerbomed }\end{array}$ & Titanium ${ }^{\star}$ & L & Cymba Concha & $\begin{array}{l}\text { Sham } \\
\text { stimulation } 25 \\
\text { Hz on ear } \\
\text { lobe }\end{array}$ & NS & $0.5 \mathrm{~mA}$ & $25 \mathrm{~Hz}$ & $30 \mathrm{~s}$ on $30 \mathrm{~s}$ off & $\begin{array}{l}\text { Inferred-tVNS improved } \\
\text { extinction learning, } \\
\text { increases in norepinephrine } \\
\text { in the prefrontal cortex and } \\
\text { limbic areas, such as the } \\
\text { amygdala and } \\
\text { hippocampus could be a } \\
\text { possible working } \\
\text { mechanism for the memory } \\
\text { enhancing effects of VNS }\end{array}$ \\
\hline Cha et al. (2016) & $\begin{array}{l}\text { Sudden-onset } \\
\text { vertigo }\end{array}$ & 1 & $\begin{array}{l}\text { ES-420, Ito } \\
\text { Company Ltd }\end{array}$ & Ball electrode & $\mathrm{R}$ & $\begin{array}{l}\text { Cymba concha, } \\
\text { cavum concha, and } \\
\text { outer surface of } \\
\text { tragus }\end{array}$ & NS & $0.2 \mathrm{~ms}$ & $\begin{array}{l}\text { Discomfort } \\
\text { threshold }\end{array}$ & $30 \mathrm{~Hz}$ & 4 min each site & $\begin{array}{l}\text { Inferred-tVNS may } \\
\text { normalize autonomic } \\
\text { imbalance due to increased } \\
\text { sympathetic response } \\
\text { causing vertigo }\end{array}$ \\
\hline $\begin{array}{l}\text { Frokaer et al. } \\
\text { (2016) }\end{array}$ & Pain threshold & 18 & $\begin{array}{l}\text { NEMOS, } \\
\text { Cerbomed }\end{array}$ & Titanium* & L & Concha & $\begin{array}{l}\text { Sham } \\
\text { stimulation } 30 \\
\text { Hz on ear } \\
\text { lobe }\end{array}$ & $0.25 \mathrm{~ms}$ & $\begin{array}{l}\text { Tingling } \\
\text { without } \\
\text { pain }\end{array}$ & $30 \mathrm{~Hz}$ & $60 \mathrm{~min}$ & NS \\
\hline $\begin{array}{l}\text { Gaul et al. } \\
\text { (2016) }\end{array}$ & $\begin{array}{l}\text { Chronic cluster } \\
\text { headache }\end{array}$ & 45 & NS & Stainless steel & $\mathrm{R}$ & Neck & NS & NS & $60 \mathrm{~mA}$ & $25 \mathrm{~Hz}$ & $\begin{array}{l}1 \mathrm{~ms} \text { on, } 40 \\
\mathrm{~ms} \text { off for three } \\
\text { doses of } 2 \mathrm{~min} \\
\text { of stimulation } \\
\text { twice a day }\end{array}$ & NS \\
\hline $\begin{array}{l}\text { Grazzi et al. } \\
(2016)\end{array}$ & $\begin{array}{l}\text { Menstrual related } \\
\text { migraine }\end{array}$ & 51 & $\begin{array}{l}\text { gammaCore } \\
\text { electroCore LLC }\end{array}$ & Stainless Steel & L, R & Neck & NS & $0.2 \mathrm{~ms}$ & $\begin{array}{l}\text { Up to } 60 \\
\mathrm{~mA}\end{array}$ & $25 \mathrm{~Hz}$ & $\begin{array}{l}\text { Burst ( } 1 \mathrm{~ms} \text { on, } \\
50 \mathrm{~ms} \text { off) for } 2 \\
\text { min three times } \\
\text { a day }\end{array}$ & NS \\
\hline $\begin{array}{l}\text { Lerman et al. } \\
\text { (2016) }\end{array}$ & $\begin{array}{l}\text { Peripheral immune } \\
\text { system } \\
\text { modulation in } \\
\text { healthy humans }\end{array}$ & 20 & $\begin{array}{l}\text { gammaCore } \\
\text { electroCore LLC }\end{array}$ & Stainless steel & L, R & Neck & $\begin{array}{l}\text { Active control } \\
1 \mathrm{~Hz} \\
\text { stimulation }\end{array}$ & $0.2 \mathrm{~ms}$ & $\begin{array}{l}\text { Tingling } \\
\text { without } \\
\text { pain }\end{array}$ & $25 \mathrm{~Hz}$ & $\begin{array}{l}\text { Burst ( } 1 \mathrm{~ms} \text { on, } \\
40 \mathrm{~ms} \text { off for } 2 \\
\text { min }\end{array}$ & NS \\
\hline
\end{tabular}


TABLE 2 | Continued

\begin{tabular}{|c|c|c|c|c|c|c|c|c|c|c|c|c|}
\hline References & Condition/Study & Participants & tVNS device & Electrode type & $\begin{array}{l}\text { Stimulation } \\
\text { Side }\end{array}$ & $\begin{array}{l}\text { Stimulation } \\
\text { Site }\end{array}$ & $\begin{array}{l}\text { Sham } \\
\text { control }\end{array}$ & $\begin{array}{l}\text { Pulse width } \\
\text { (ms) }\end{array}$ & $\begin{array}{l}\text { Intensity } \\
\text { (mA) }\end{array}$ & Freq $(\mathrm{Hz})$ & $\begin{array}{l}\text { Duty } \\
\text { cycle/Time }\end{array}$ & Brain activation \\
\hline $\begin{array}{l}\text { Rong et al. } \\
\text { (2016) }\end{array}$ & $\begin{array}{l}\text { Major depressive } \\
\text { disorder }\end{array}$ & 160 & NS & Ear clips & NS & Concha & $\begin{array}{l}\text { Sham } \\
\text { stimulation } 20 \\
\mathrm{~Hz} \text { at superior } \\
\text { scapha }\end{array}$ & $0.2 \mathrm{~ms}$ & $\begin{array}{l}\text { Tolerance } \\
\text { threshold } \\
\text { (typically } \\
\text { between } 4 \\
\text { and } 6 \mathrm{~mA} \text { ) }\end{array}$ & $20 \mathrm{~Hz}$ & $\begin{array}{l}\text { Continuously } \\
\text { for } 30 \text { min } \\
\text { twice a day }\end{array}$ & NS \\
\hline $\begin{array}{l}\text { Silberstein et al. } \\
\text { (2016a) }\end{array}$ & Migraine & 59 & $\begin{array}{l}\text { gammaCore } \\
\text { electroCore LLC }\end{array}$ & Stainless steel & $\mathrm{R}$ & Neck & $\begin{array}{l}\text { Sham device } \\
\text { that did not } \\
\text { deliver } \\
\text { electrical } \\
\text { stimulation }\end{array}$ & NS & $\begin{array}{l}\text { Set by the } \\
\text { user (up to } \\
60 \mathrm{~mA} \text { ) }\end{array}$ & NS & $\begin{array}{l}2 \times 2 \text { min } \\
\text { doses } \\
\text { delivered } 5-10 \\
\text { min apart three } \\
\text { times a day }\end{array}$ & NS \\
\hline $\begin{array}{l}\text { Silberstein et al. } \\
\text { (2016b) }\end{array}$ & Cluster headache & 150 & $\begin{array}{l}\text { gammaCore } \\
\text { electroCore LLC }\end{array}$ & Stainless steel & $\mathrm{R}$ & Neck & $\begin{array}{l}\text { Sham device } \\
\text { delivering } 0.1 \\
\text { Hz biphasic } \\
\text { pulse }\end{array}$ & $0.2 \mathrm{~ms}$ & $\begin{array}{l}\text { Set by the } \\
\text { user (up to } \\
60 \mathrm{~mA} \text { ) }\end{array}$ & $25 \mathrm{~Hz}$ & $\begin{array}{l}\text { Burst ( } 1 \mathrm{~ms} \text { on, } \\
40 \text { ms off) for } \\
\text { three } \\
\text { consecutive } 2 \\
\text { min } \\
\text { stimulations } 1 \\
\text { min apart }\end{array}$ & $\begin{array}{l}\text { Inferred-stimulation of } \\
\text { vagus nerve affects } \\
\text { hypocretin and orexin } \\
\text { pathway that affects } \\
\text { pathophysiology of cluster } \\
\text { headaches }\end{array}$ \\
\hline $\begin{array}{l}\text { Trevizol et al. } \\
\text { (2016) }\end{array}$ & Depression & 12 & $\begin{array}{l}\text { Ibramed } \\
\text { Neurodyn II }\end{array}$ & Rubber electrodes & $L, R$ & Mastoid process & NS & $0.25 \mathrm{~ms}$ & $12 \mathrm{~mA}$ & $120 \mathrm{~Hz}$ & $\begin{array}{l}30 \text { min a day } \\
10 \text { times over } 2 \\
\text { weeks }\end{array}$ & NS \\
\hline $\begin{array}{l}\text { Fang et al. } \\
\text { (2016) }\end{array}$ & $\begin{array}{l}\text { Major depressive } \\
\text { disorder }\end{array}$ & 34 & NS & Ear clip & L & Concha & $\begin{array}{l}\text { Sham } \\
\text { stimulation } 20 \\
\mathrm{~Hz} \text { at superior } \\
\text { scapha }\end{array}$ & $<1 \mathrm{~ms}$ & $\begin{array}{l}\text { Tolerance } \\
\text { threshold } \\
(4-6 \mathrm{~mA})\end{array}$ & $20 \mathrm{~Hz}$ & $\begin{array}{l}2 \times 30 \text { min } \\
\text { daily, } 5 \text { days a } \\
\text { week for } 4 \\
\text { weeks }\end{array}$ & $\begin{array}{l}\text { Measured-fMRI showed } \\
\text { that after tVNS default } \\
\text { mode network functional } \\
\text { connectivity showed } \\
\text { significant changes in brain } \\
\text { regions involved in } \\
\text { emotional modulation } \\
\text { which is associated with } \\
\text { depression severity }\end{array}$ \\
\hline $\begin{array}{l}\text { Frangos et al. } \\
\text { (2015) }\end{array}$ & $\begin{array}{l}\text { Bold fMRI effects } \\
\text { of tVNS }\end{array}$ & 12 & $\begin{array}{l}\text { NEMOS, } \\
\text { Cerbomed }\end{array}$ & Titanium & L & Cymba Concha & $\begin{array}{l}\text { Sham } \\
\text { stimulation } 25 \\
\text { Hz on ear } \\
\text { lobe }\end{array}$ & $0.25 \mathrm{~ms}$ & $\begin{array}{l}\text { Tingling } \\
\text { but not } \\
\text { painful } \\
(0.3-0.8 \\
\mathrm{mA})\end{array}$ & $25 \mathrm{HZ}$ & $\begin{array}{l}\text { Continuously } \\
\text { for } 14 \text { min }\end{array}$ & $\begin{array}{l}\text { Measured-fMRI shows } \\
\text { tVNS significantly affects } \\
\text { central projections of the } \\
\text { vagus nerve. }\end{array}$ \\
\hline $\begin{array}{l}\text { Hyvärinen et al. } \\
\text { (2015) }\end{array}$ & Tinnitus & 15 & Tinnoff Inc & Clip electrode & L & Tragus & $\begin{array}{l}\text { Sham } \\
\text { stimulation } 25 \\
\text { Hz on ear } \\
\text { lobe }\end{array}$ & $0.5 \mathrm{~ms}$ & $\begin{array}{l}\text { Above } \\
\text { sensory } \\
\text { threshold } \\
(\sim 0.5 \mathrm{~mA})\end{array}$ & $25 \mathrm{~Hz}$ & $\begin{array}{l}\text { Continuously } \\
\text { for } 6 \text { min }\end{array}$ & $\begin{array}{l}\text { Measured-MEG showed } \\
\text { tVNS modulates synchrony } \\
\text { of tone-evoked brain } \\
\text { activity, especially at the } \\
\text { beta and gamma bands }\end{array}$ \\
\hline $\begin{array}{l}\text { Nesbitt et al. } \\
\text { (2015) }\end{array}$ & Cluster headache & 19 & $\begin{array}{l}\text { gammaCore } \\
\text { electroCore LLC }\end{array}$ & Stainless stee|* & $L, R$ & Neck & NS & $1 \mathrm{~ms}$ & $\begin{array}{l}\text { Self- } \\
\text { controlled }\end{array}$ & $25 \mathrm{~Hz}$ & $\begin{array}{l}2 \text { min per } \\
\text { dose, up to } \\
\text { three doses } \\
\text { twice daily }\end{array}$ & NS \\
\hline $\begin{array}{l}\text { Sellaro et al. } \\
\text { (2015b) }\end{array}$ & Post-error slowing & 40 & $\begin{array}{l}\text { CM02, } \\
\text { Cerbomed }\end{array}$ & Two titan electrodes & L & Outer auditory canal & $\begin{array}{l}\text { Sham } \\
\text { stimulation } 25 \\
\text { Hz on ear } \\
\text { lobe }\end{array}$ & $0.2-0.3 \mathrm{~ms}$ & $0.5 \mathrm{~mA}$ & $25 \mathrm{~Hz}$ & $\begin{array}{l}30 \mathrm{~s} \text { on and } 30 \\
\mathrm{~s} \text { off for } 75 \mathrm{~min}\end{array}$ & NS \\
\hline $\begin{array}{l}\text { Sellaro et al. } \\
\text { (2015a) }\end{array}$ & $\begin{array}{l}\text { Pro-social } \\
\text { behavior }\end{array}$ & 24 & $\begin{array}{l}\text { CM02, } \\
\text { Cerbomed }\end{array}$ & Two titan electrodes & L & Outer auditory canal & $\begin{array}{l}\text { Sham } \\
\text { stimulation } 25 \\
\text { Hz on ear } \\
\text { lobe }\end{array}$ & $0.2-0.3 \mathrm{~ms}$ & $0.5 \mathrm{~mA}$ & $25 \mathrm{~Hz}$ & $\begin{array}{l}30 \mathrm{~s} \text { on and } 30 \\
\mathrm{~s} \text { off for } 26 \mathrm{~min}\end{array}$ & $\begin{array}{l}\text { Inferred-tVNS expected to } \\
\text { enhance prosocial helping } \\
\text { behavior due to activation } \\
\text { in the insula and prefrontal } \\
\text { cortex but this was not } \\
\text { observed }\end{array}$ \\
\hline
\end{tabular}


TABLE 2 | Continued

\begin{tabular}{|c|c|c|c|c|c|c|c|c|c|c|c|c|}
\hline References & Condition/Study & Participants & tVNS device & Electrode type & $\begin{array}{l}\text { Stimulation } \\
\text { Side }\end{array}$ & $\begin{array}{l}\text { Stimulation } \\
\text { Site }\end{array}$ & $\begin{array}{l}\text { Sham } \\
\text { control }\end{array}$ & $\begin{array}{l}\text { Pulse width } \\
\text { (ms) }\end{array}$ & $\begin{array}{l}\text { Intensity } \\
(\mathrm{mA})\end{array}$ & Freq $(\mathrm{Hz})$ & $\begin{array}{l}\text { Duty } \\
\text { cycle/Time }\end{array}$ & Brain activation \\
\hline $\begin{array}{l}\text { Altavilla et al. } \\
\text { (2015) }\end{array}$ & Migraine & 20 & $\begin{array}{l}\text { gammaCore } \\
\text { electroCore LLC }\end{array}$ & Stainless steel $\left.\right|^{*}$ & NS & Neck & NS & NS & NS & NS & $\begin{array}{l}\text { Continuously } \\
\text { for } 90 \text { s }\end{array}$ & NS \\
\hline $\begin{array}{l}\text { Barbanti et al. } \\
\text { (2015) }\end{array}$ & Chronic Migraine & 50 & $\begin{array}{l}\text { gammaCore } \\
\text { electroCore LLC }\end{array}$ & Stainless steel $\left.\right|^{*}$ & $\mathrm{R}$ & Neck & NS & NS & NS & NS & $\begin{array}{l}2 \times 120 \mathrm{~s} \\
\text { doses } 3 \mathrm{~min} \\
\text { apart per } \\
\text { migraine }\end{array}$ & NS \\
\hline $\begin{array}{l}\text { Hasan et al. } \\
\text { (2015) }\end{array}$ & Schizophrenia & 20 & $\begin{array}{l}\text { CM02, } \\
\text { Cerbomed }\end{array}$ & Two titan electrodes & L & Outer auditory canal & $\begin{array}{l}\text { No electrical } \\
\text { stimulation } \\
\text { delivered }\end{array}$ & $0.25 \mathrm{~ms}$ & $\begin{array}{l}\text { Above } \\
\text { perception } \\
\text { threshold }\end{array}$ & $25 \mathrm{~Hz}$ & $\begin{array}{l}30 \mathrm{~s} \text { on, } 180 \mathrm{~s} \\
\text { off for up to } 3 \\
\times 3 \mathrm{~h} \text { a day }\end{array}$ & NS \\
\hline $\begin{array}{l}\text { Jacobs et al. } \\
\text { (2015) }\end{array}$ & $\begin{array}{l}\text { Associative } \\
\text { memory in older } \\
\text { individuals }\end{array}$ & 30 & $\begin{array}{l}\text { TENSTem } \\
\text { dental, } \\
\text { Schwa-medico } \\
\text { BV }\end{array}$ & Circular ear clip & L & $\begin{array}{l}\text { External acoustic } \\
\text { meatus on inner side } \\
\text { of tragus }\end{array}$ & $\begin{array}{l}\text { No electrical } \\
\text { stimulation } \\
\text { delivered }\end{array}$ & $0.2 \mathrm{~ms}$ & $5 \mathrm{~mA}$ & $8 \mathrm{~Hz}$ & Twice a day & $\begin{array}{l}\text { Inferred-tVNS enhances } \\
\text { memory performance by } \\
\text { increasing locus coeruleus } \\
\text { activity and noradrenalin } \\
\text { levels to memory-relevant } \\
\text { brain areas. }\end{array}$ \\
\hline $\begin{array}{l}\text { Kinfe et al. } \\
\text { (2015a) }\end{array}$ & $\begin{array}{l}\text { Cluster-Tic } \\
\text { syndrome }\end{array}$ & 1 & $\begin{array}{l}\text { gammaCore } \\
\text { electroCore LLC }\end{array}$ & Stainless steel ${ }^{*}$ & $\mathrm{R}$ & Neck & NS & $1 \mathrm{~ms}$ & $12-14 \mathrm{~V}$ & $25 \mathrm{~Hz}$ & $\begin{array}{l}\text { Burst for } 2 \times \\
90 \text { s doses } 15 \\
\text { min apart }\end{array}$ & NS \\
\hline $\begin{array}{l}\text { Kinfe et al. } \\
(2015 b)\end{array}$ & $\begin{array}{l}\text { Migraine and } \\
\text { sleep disturbance }\end{array}$ & 20 & $\begin{array}{l}\text { gammaCore } \\
\text { electroCore LLC }\end{array}$ & Stainless steel $\left.\right|^{*}$ & $L, R$ & Neck & NS & $1 \mathrm{~ms}$ & $0-24 \mathrm{~V}$ & $25 \mathrm{~Hz}$ & $\begin{array}{l}\text { Burst for } 2 \times 2 \\
\text { min twice a day }\end{array}$ & $\begin{array}{l}\text { Inferred-in patients with } \\
\text { migraine, and tVNS may } \\
\text { help to counteract the } \\
\text { decline in thalamocortical } \\
\text { activity }\end{array}$ \\
\hline $\begin{array}{l}\text { Stavrakis et al. } \\
\text { (2015) }\end{array}$ & Atrial fibrillation & 40 & $\begin{array}{l}\text { Grass S88, } \\
\text { Natus } \\
\text { Neurology Inc }\end{array}$ & Flat metal clip & $\mathrm{R}$ & Tragus & $\begin{array}{l}\text { No electrical } \\
\text { stimulation } \\
\text { delivered }\end{array}$ & $1 \mathrm{~ms}$ & $\begin{array}{l}\text { Discomfort } \\
\text { threshold }\end{array}$ & $20 \mathrm{~Hz}$ & $\begin{array}{l}\text { Continuously } \\
\text { for } 60 \text { min } \\
\text { following } \\
\text { induction of } \\
\text { atrial fibrillation }\end{array}$ & NS \\
\hline $\begin{array}{l}\text { Steenbergen } \\
\text { et al. (2015) }\end{array}$ & $\begin{array}{l}\text { Efficiency of action } \\
\text { cascading } \\
\text { processes in } \\
\text { healthy humans }\end{array}$ & 30 & $\begin{array}{l}\text { CM02, } \\
\text { Cerbomed }\end{array}$ & Two titan electrodes & L & Outer auditory canal & $\begin{array}{l}\text { Sham } \\
\text { stimulation } 25 \\
\text { Hz on ear } \\
\text { lobe }\end{array}$ & $0.2-0.3 \mathrm{~ms}$ & $0.5 \mathrm{~mA}$ & $25 \mathrm{~Hz}$ & $\begin{array}{l}30 \mathrm{~s} \text { on, } 30 \mathrm{~s} \\
\text { off for } 45 \mathrm{~min}\end{array}$ & $\begin{array}{l}\text { Inferred-tVNS modulates } \\
\text { efficiency of action } \\
\text { cascading processes, likely } \\
\text { via GABA and NE release }\end{array}$ \\
\hline $\begin{array}{l}\text { Straube et al. } \\
\text { (2015) }\end{array}$ & Migraine & 46 & $\begin{array}{l}\text { NEMOS, } \\
\text { Cerbomed }\end{array}$ & Titanium $^{*}$ & L & Concha & $\begin{array}{l}\text { Active control } \\
1 \mathrm{~Hz} \text { sham } \\
\text { stimulation }\end{array}$ & $0.25 \mathrm{~ms}$ & $\begin{array}{l}\text { Tingling } \\
\text { but not } \\
\text { painful }\end{array}$ & 1 or $25 \mathrm{~Hz}$ & $\begin{array}{l}30 \mathrm{~s} \text { on, } 30 \mathrm{~s} \\
\text { off for } 4 \mathrm{~h} \text { a day } \\
\text { for } 12 \text { weeks }\end{array}$ & $\begin{array}{l}\text { Inferred-headache } \\
\text { decreased more } \\
\text { significantly in } 1 \mathrm{~Hz} \text { active } \\
\text { control group, possibly due } \\
\text { to suppression of } \\
\text { nociceptive signaling and } \\
\text { pain perception in spinal } \\
\text { trigeminal nucleus.tVSS } \\
\text { may also alter cortical } \\
\text { excitability }\end{array}$ \\
\hline $\begin{array}{l}\text { Weise et al. } \\
\text { (2015) }\end{array}$ & $\begin{array}{l}\text { Parkinson's } \\
\text { disease }\end{array}$ & 50 & NS & $\begin{array}{l}\text { Custom made fine } \\
\text { silver wires }\end{array}$ & $L, R$ & Tragus & NS & $0.1 \mathrm{~ms}$ & $8 \mathrm{~mA}$ & $0.5 \mathrm{~Hz}$ & NS & $\begin{array}{l}\text { Measured-scalp } \\
\text { electrodes measured } \\
\text { activation of brainstem after } \\
\text { tVNS and observed } \\
\text { somatosensory evoked } \\
\text { potentials in the nerve } \\
\text { which is believed to reflect } \\
\text { neuronal activity }\end{array}$ \\
\hline Mei et al. (2014) & Tinnitus & 32 & $\begin{array}{l}\text { TENS-200, } \\
\text { Suzhou Medical } \\
\text { Supplies Co Ltd }\end{array}$ & NS & NS & Cavum Concha & NS & $1 \mathrm{~ms}$ & $1 \mathrm{~mA}$ & $20 \mathrm{~Hz}$ & $\begin{array}{l}2 \times 20 \text { min } \\
\text { daily for } 8 \\
\text { weeks }\end{array}$ & NS \\
\hline
\end{tabular}




\begin{tabular}{|c|c|c|c|c|c|c|c|c|c|c|c|c|}
\hline References & Condition/Study & Participants & tVNS device & Electrode type & $\begin{array}{l}\text { Stimulation } \\
\text { Side }\end{array}$ & $\begin{array}{l}\text { Stimulation } \\
\text { Site }\end{array}$ & $\begin{array}{l}\text { Sham } \\
\text { control }\end{array}$ & $\begin{array}{l}\text { Pulse width } \\
\text { (ms) }\end{array}$ & $\begin{array}{l}\text { Intensity } \\
(\mathrm{mA})\end{array}$ & Freq $(\mathrm{Hz})$ & $\begin{array}{l}\text { Duty } \\
\text { cycle/Time }\end{array}$ & Brain activation \\
\hline $\begin{array}{l}\text { Ainua et al. } \\
\text { (2014) }\end{array}$ & Epilepsy & 60 & TENS-200 & NS & L, R & $\begin{array}{l}\text { Outer auditory canal } \\
\text { and conchal cavity }\end{array}$ & $\begin{array}{l}\text { Sham } \\
\text { stimulation } 20 \\
\mathrm{~Hz} \text { on ear } \\
\text { lobe }\end{array}$ & $0.2 \mathrm{~ms}$ & $\begin{array}{l}\text { Individual } \\
\text { specific }\end{array}$ & $20 \mathrm{~Hz}$ & $\begin{array}{l}\text { Continuously } \\
\text { for } 20 \text { min } \\
\text { three times a } \\
\text { day }\end{array}$ & NS \\
\hline $\begin{array}{l}\text { Capone et al. } \\
\text { (2015) }\end{array}$ & $\begin{array}{l}\text { Cortical excitability } \\
\text { in healthy } \\
\text { volunteers }\end{array}$ & 10 & Twister, EBM & $\mathrm{Ag} / \mathrm{AgCl}$ & L & $\begin{array}{l}\text { External acoustic } \\
\text { meatus at inner side } \\
\text { of tragus }\end{array}$ & $\begin{array}{l}\text { Sham } \\
\text { stimulation } 20 \\
\text { Hz on ear } \\
\text { lobe }\end{array}$ & $0.3 \mathrm{~ms}$ & $8 \mathrm{~mA}$ & $20 \mathrm{~Hz}$ & $\begin{array}{l}30 \mathrm{~s} \text { on, } 270 \mathrm{~s} \\
\text { off for } 1 \mathrm{~h}\end{array}$ & $\begin{array}{l}\text { Measured-measurement } \\
\text { of motor evoked potentials } \\
\text { showed a GABA } \\
\text { modulation in the motor } \\
\text { cortex contralateral to the } \\
\text { tVNS stimulation side }\end{array}$ \\
\hline $\begin{array}{l}\text { Clancy et al. } \\
\text { (2014) }\end{array}$ & $\begin{array}{l}\text { Sympathetic nerve } \\
\text { activity in healthy } \\
\text { humans }\end{array}$ & 48 & $\begin{array}{l}\text { V-TENS PLUS, } \\
\text { Body Clock } \\
\text { Health Care Ltd }\end{array}$ & $\begin{array}{l}\text { Modified surface } \\
\text { electrodes }\end{array}$ & NS & Tragus & $\begin{array}{l}\text { Disconnected } \\
\text { electrodes for } \\
\text { sham }\end{array}$ & $0.2 \mathrm{~ms}$ & $\begin{array}{l}\text { Sensory } \\
\text { threshold } \\
(10-50 \\
\text { mA) }\end{array}$ & $30 \mathrm{~Hz}$ & $\begin{array}{l}\text { Continuously } \\
\text { for } 15 \text { min }\end{array}$ & NS \\
\hline $\begin{array}{l}\text { Goadsby et al. } \\
\text { (2014) }\end{array}$ & Acute Migraine & 30 & $\begin{array}{l}\text { gammaCore } \\
\text { electroCore LLC }\end{array}$ & Stainless steel $\left.\right|^{*}$ & $\mathrm{R}$ & Neck & NS & NS & NS & NS & $\begin{array}{l}2 \times 90 \text { s doses } \\
15 \text { min apart } \\
\text { after migraine } \\
\text { onset }\end{array}$ & NS \\
\hline $\begin{array}{l}\text { Grazzi et al. } \\
(2014)\end{array}$ & Migraine & 30 & $\begin{array}{l}\text { gammaCore } \\
\text { electroCore LLC }\end{array}$ & Stainless stee|* & $\mathrm{R}$ & Neck & NS & NS & NS & NS & $90 \mathrm{~s}$ & NS \\
\hline $\begin{array}{l}\text { Huang et al. } \\
\text { (2014) }\end{array}$ & $\begin{array}{l}\text { Impaired glucose } \\
\text { tolerance }\end{array}$ & 72 & $\begin{array}{l}\text { Huatuo } \\
\text { TENS-200, } \\
\text { Suzhou }\end{array}$ & NS & NS & Concha & $\begin{array}{l}\text { Sham } \\
\text { stimulation } 20 \\
\mathrm{~Hz} \text { applied at } \\
\text { superior } \\
\text { scapha }\end{array}$ & $=1 \mathrm{~ms}$ & $\begin{array}{l}1.0 \\
\text { (adjusted } \\
\text { based on } \\
\text { tolerance) }\end{array}$ & $20 \mathrm{~Hz}$ & $\begin{array}{l}20 \text { min twice } \\
\text { daily for } 12 \\
\text { weeks }\end{array}$ & NS \\
\hline $\begin{array}{l}\text { Kreuzer et al. } \\
\text { (2014) }\end{array}$ & Tinnitus & 50 & $\begin{array}{l}\text { Phase I: CMO2, } \\
\text { Cerbomed } \\
\text { Phase II: } \\
\text { NEMOS, } \\
\text { Cerbomed }\end{array}$ & Two titan electrodes & NS & NS & NS & NS & $0.1-10 \mathrm{~mA}$ & $25 \mathrm{~Hz}$ & $\begin{array}{l}\text { Phase l: } 30 \mathrm{~s} \\
\text { on, } 180 \mathrm{~s} \text { off } \\
\text { for } 6 \mathrm{~h} \text { per day } \\
\text { Phase Il: } 30 \mathrm{~s} \\
\text { on, } 30 \mathrm{~s} \text { off for } \\
4 \mathrm{~h} \text { per day }\end{array}$ & NS \\
\hline $\begin{array}{l}\text { Laqua et al. } \\
\text { (2014) }\end{array}$ & $\begin{array}{l}\text { Pain threshold in } \\
\text { healthy humans }\end{array}$ & 22 & $\begin{array}{l}\text { TNS SM } 2 \text { MF, } \\
\text { Schwamedico } \\
\text { GmbH }\end{array}$ & $\begin{array}{l}\text { Anode: Silver disc } \\
\text { Cathode: PECG } \\
\text { electrode }\end{array}$ & $L, R$ & $\begin{array}{l}\text { Cavum Concha and } \\
\text { Mastoid area }\end{array}$ & $\begin{array}{l}\text { No electrical } \\
\text { stimulation } \\
\text { delivered }\end{array}$ & $0.2 \mathrm{~ms}$ & $\begin{array}{l}\text { Perception } \\
\text { threshold }\end{array}$ & 2 and $100 \mathrm{~Hz}$ & Burst 30 min & $\begin{array}{l}\text { Inferred-tVNS produces } \\
\text { both anti- and } \\
\text { pro-nociceptive effects }\end{array}$ \\
\hline $\begin{array}{l}\text { Busch et al. } \\
\text { (2013) }\end{array}$ & $\begin{array}{l}\text { Pain perception in } \\
\text { healthy volunteers }\end{array}$ & 48 & $\begin{array}{l}\text { STV02, } \\
\text { Cerbomed }\end{array}$ & Bipolar electrode & L & $\begin{array}{l}\text { Concha at inner side } \\
\text { of tragus }\end{array}$ & $\begin{array}{l}\text { No electrical } \\
\text { stimulation } \\
\text { delivered }\end{array}$ & $0.25 \mathrm{~ms}$ & $\begin{array}{l}0.25-10 \\
m A\end{array}$ & $25 \mathrm{~Hz}$ & $\begin{array}{l}\text { Continuously } \\
\text { for } 1 \mathrm{~h}\end{array}$ & $\begin{array}{l}\text { Inferred-detailed analysis } \\
\text { of different sub modalities } \\
\text { of the somatosensory } \\
\text { system suggest an impact } \\
\text { of t-VNS on central pain } \\
\text { processing rather than on } \\
\text { peripheral nociceptor } \\
\text { activity }\end{array}$ \\
\hline He et al. (2013) & Pediatric epilepsy & 14 & TENS-200 & Conductive rubber & $L, R$ & Concha & NS & NS & $\begin{array}{l}0.4-1.0 \\
\mathrm{~mA} \\
\text { depending } \\
\text { on } \\
\text { tolerance }\end{array}$ & $20 \mathrm{~Hz}$ & $\begin{array}{l}3 \times 30 \mathrm{~min} \text { a } \\
\text { day }\end{array}$ & $\begin{array}{l}\text { Inferred-afferent } \\
\text { projections from the ABVN } \\
\text { to the nucleus tractus } \\
\text { solitarius rather than to the } \\
\text { spinal trigeminal nucleus } \\
\text { may explain anti-seizure } \\
\text { effect }\end{array}$ \\
\hline $\begin{array}{l}\text { Lehtimäki et al. } \\
\text { (2013) }\end{array}$ & Tinnitus & 10 & $\begin{array}{l}\text { Tinoff pulse } \\
\text { generator }\end{array}$ & Clip electrode & L & Tragus & $\begin{array}{l}\text { No electrical } \\
\text { stimulation } \\
\text { delivered }\end{array}$ & NS & $\begin{array}{l}\text { Above } \\
\text { sensory } \\
\text { threshold } \\
\text { (usually } \\
\text { around } 0.8 \\
\mathrm{~mA} \text { ) }\end{array}$ & $25 \mathrm{~Hz}$ & $\begin{array}{l}7 \times 45 / 60 \text { min } \\
\text { sessions } \\
\text { delivered over } \\
10 \text { days }\end{array}$ & $\begin{array}{l}\text { Measured-MEG shows } \\
\text { tVNS can modulate } \\
\text { auditory cortical activation }\end{array}$ \\
\hline
\end{tabular}


TABLE 2 | Continued

\begin{tabular}{|c|c|c|c|c|c|c|c|c|c|c|c|c|}
\hline References & Condition/Study & Participants & tVNS device & Electrode type & $\begin{array}{l}\text { Stimulation } \\
\text { Side }\end{array}$ & $\begin{array}{l}\text { Stimulation } \\
\text { Site }\end{array}$ & $\begin{array}{l}\text { Sham } \\
\text { control }\end{array}$ & $\begin{array}{l}\text { Pulse width } \\
\text { (ms) }\end{array}$ & $\begin{array}{l}\text { Intensity } \\
(\mathrm{mA})\end{array}$ & Freq $(\mathrm{Hz})$ & $\begin{array}{l}\text { Duty } \\
\text { cycle/Time }\end{array}$ & Brain activation \\
\hline $\begin{array}{l}\text { Kraus et al. } \\
\text { (2013) }\end{array}$ & $\begin{array}{l}\text { Effects of } \\
\text { sham-controlled } \\
\text { transcutaneous } \\
\text { electrical } \\
\text { stimulation }\end{array}$ & 16 & Digitimer DS7A & Silver & L & $\begin{array}{l}\text { Group I: Anterior wall } \\
\text { of ear canal Group II: } \\
\text { posterior side of ear } \\
\text { canal }\end{array}$ & $\begin{array}{l}\text { Sham } \\
\text { stimulation } 8 \\
\text { Hz on ear } \\
\text { lobe }\end{array}$ & $0.02 \mathrm{~ms}$ & $\begin{array}{l}\text { Non- } \\
\text { painful }\end{array}$ & $8 \mathrm{~Hz}$ & $\begin{array}{l}4 \times 30 \text { s on, } \\
60 \text { s off }\end{array}$ & $\begin{array}{l}\text { Measured-fMRI shows } \\
\text { activations and } \\
\text { deactivations of certain } \\
\text { brain regions, especially } \\
\text { frontal and limbic areas } \\
\text { depending on area of } \\
\text { stimulation, and showed } \\
\text { more activation than in } \\
\text { sham stimulation }\end{array}$ \\
\hline $\begin{array}{l}\text { Hein et al. } \\
\text { (2012) }\end{array}$ & Depression & 37 & $\begin{array}{l}\text { Study1: } \\
\text { TENS-NET } \\
\text { 2000, Auri-Stim } \\
\text { Medical Inc } \\
\text { Study 2: } \\
\text { TENS-NET } \\
\text { 1000, Auri-Stim } \\
\text { Medical Inc }\end{array}$ & $\begin{array}{l}\text { Headset (4 electrodes } \\
\text { placed crosswise) }\end{array}$ & L, R & Outer auditory canal & $\begin{array}{l}\text { No electrical } \\
\text { stimulation } \\
\text { delivered } \\
\text { electrodes } \\
\text { unplugged }\end{array}$ & NS & $\begin{array}{l}\text { Study 1: } \\
\text { Perception } \\
\text { threshold } \\
\text { Study 2: } \\
130 \mu \mathrm{A}\end{array}$ & $1.5 \mathrm{~Hz}$ & $\begin{array}{l}\text { Study } 1: 1 \times \\
15 \text { min } 5 \text { days } \\
\text { a week Study } \\
2: 2 \times 15 \text { min } \\
5 \text { days a week }\end{array}$ & NS \\
\hline $\begin{array}{l}\text { Napadow et al. } \\
\text { (2012) }\end{array}$ & $\begin{array}{l}\text { Chronic pelvic } \\
\text { pain }\end{array}$ & 15 & $\begin{array}{l}\text { Cefar Acus II, } \\
\text { Cefar Medical }\end{array}$ & $\begin{array}{l}\text { Modified press-tack } \\
\text { electrode }\end{array}$ & L & $\begin{array}{l}\text { Cymba Concha and } \\
\text { slope between } \\
\text { antihelix and cavum } \\
\text { concha }\end{array}$ & $\begin{array}{l}\text { Sham } \\
\text { stimulation } 30 \\
\text { Hz on ear } \\
\text { lobe }\end{array}$ & $0.45 \mathrm{~ms}$ & $\begin{array}{l}\text { Strong, } \\
\text { non- } \\
\text { painful }\end{array}$ & $30 \mathrm{~Hz}$ & $\begin{array}{l}0.5 \mathrm{~s} \text { on, } \\
\text { matched to } \\
\text { respiration for } \\
30 \mathrm{~min}\end{array}$ & NS \\
\hline $\begin{array}{l}\text { Stefan et al. } \\
\text { (2012) }\end{array}$ & Epilepsy & 10 & NS & NS & L & Tragus & NS & $0.3 \mathrm{~ms}$ & $\begin{array}{l}\text { Tolerance } \\
\text { threshold }\end{array}$ & $10 \mathrm{~Hz}$ & $\begin{array}{l}3 \times 1 \mathrm{~h} \text { a day } \\
\text { over } 9 \text { months }\end{array}$ & NS \\
\hline $\begin{array}{l}\text { Schulz-Stübner } \\
\text { and Kehl (2011) }\end{array}$ & Hiccups & 1 & $\begin{array}{l}\text { NMS 300, } \\
\text { Xavant } \\
\text { Technology }\end{array}$ & NS & L & Neck & NS & NS & $6 \mathrm{~mA}$ & $1 \mathrm{~Hz}$ & $30 \mathrm{~s}$ & $\begin{array}{l}\text { Inferred-Unclear whether } \\
\text { hiccups were stopped due } \\
\text { to interference with reflex } \\
\text { arches at different neuronal } \\
\text { levels }\end{array}$ \\
\hline $\begin{array}{l}\text { Dietrich et al. } \\
\text { (2008) }\end{array}$ & Bold fMRI & 4 & Cerbomed & Silver & L & Tragus & NS & $0.25 \mathrm{~ms}$ & $4-8 \mathrm{~mA}$ & $25 \mathrm{~Hz}$ & $\begin{array}{l}50 \mathrm{~s} \text { on, } 100 \mathrm{~s} \\
\text { off for } 700 \mathrm{~s}\end{array}$ & $\begin{array}{l}\text { Measured-Bold fMRI } \\
\text { showed tVNS elicited a } \\
\text { robust activation in the left } \\
\text { locus coeruleus, a } \\
\text { brainstem nucleus related } \\
\text { to clinical depression as } \\
\text { well as bilateral activation } \\
\text { of the thalamus }\end{array}$ \\
\hline $\begin{array}{l}\text { Kraus et al. } \\
(2007)\end{array}$ & Bold fMRI & 22 & $\begin{array}{l}\text { EMP2 Expert, } \\
\text { Schwa-medico } \\
\text { GmbH }\end{array}$ & Silver & L & Tragus & $\begin{array}{l}\text { Sham } \\
\text { stimulation } 8 \\
\text { Hz on ear } \\
\text { lobe }\end{array}$ & $0.02 \mathrm{~ms}$ & $\begin{array}{l}\text { Perception } \\
\text { threshold }\end{array}$ & $8 \mathrm{~Hz}$ & $\begin{array}{l}30 \mathrm{~s} \text { on, } 120 \mathrm{~s} \\
\text { off three times } \\
\text { over } 2 \text { days }\end{array}$ & $\begin{array}{l}\text { Measured-fMRI shows } \\
\text { tVNS leads to prominent } \\
\text { changes in cerebral } \\
\text { activation patterns, with } \\
\text { marked deactivation in } \\
\text { limbic and temporal brain } \\
\text { areas }\end{array}$ \\
\hline $\begin{array}{l}\text { Fallgatter et al. } \\
\text { (2003) }\end{array}$ & $\begin{array}{l}\text { Vagus sensory } \\
\text { evoked potentials }\end{array}$ & 6 & NS & Bipolar electrode & NS & $\begin{array}{l}\text { Tragus and acoustic } \\
\text { meatus }\end{array}$ & NS & $0.1 \mathrm{~ms}$ & $8 \mathrm{~mA}$ & NS & $\begin{array}{l}2 \mathrm{~s} \\
\text { interstimulus } \\
\text { interval }\end{array}$ & $\begin{array}{l}\text { Measured-Evoked } \\
\text { potential recordings are far } \\
\text { field potentials of } \\
\text { post-synaptic brainstem } \\
\text { activity from vagus nerve } \\
\text { nuclei that can be elicited } \\
\text { on electrical stimulation }\end{array}$ \\
\hline $\begin{array}{l}\text { Johnson et al. } \\
\text { (1991) }\end{array}$ & $\begin{array}{l}\text { Pain threshold and } \\
\text { autonomic } \\
\text { function }\end{array}$ & 24 & Microtens 7757 & $\mathrm{Ag} / \mathrm{AgCl}$ and rubber & $\mathrm{R}$ & Concha & $\begin{array}{l}\text { No electrical } \\
\text { stimulation } \\
\text { delivered }\end{array}$ & $0.5 \mathrm{~ms}$ & $\begin{array}{l}\text { Discomfort } \\
\text { threshold }\end{array}$ & $2.3 \mathrm{~Hz}$ & $\begin{array}{l}\text { Burst for } 15 \\
\text { min }\end{array}$ & NS \\
\hline
\end{tabular}

NS, not stated. An asterisk indicates that an electrode type was not stated in the study but was assumed by us from the type of the device. A dagger indicates parameters as stated in the original paper but that are outside the normal range (possible typing error). 


\section{A}

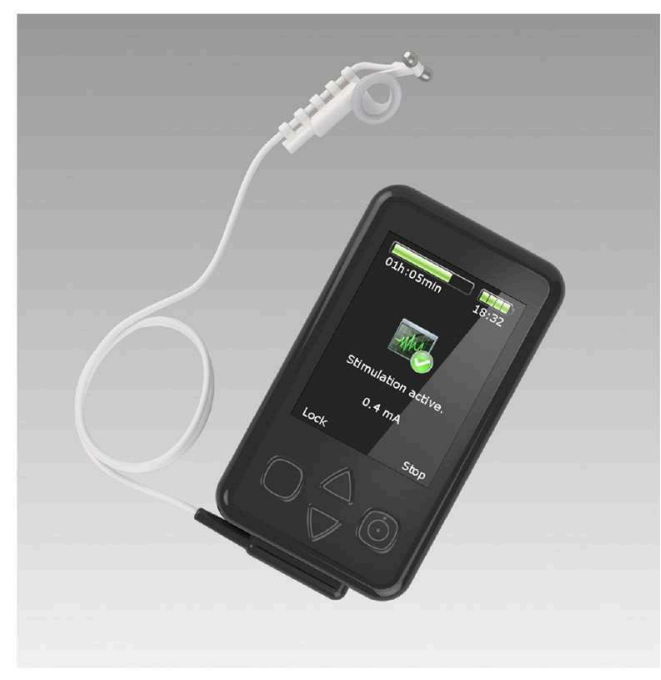

B

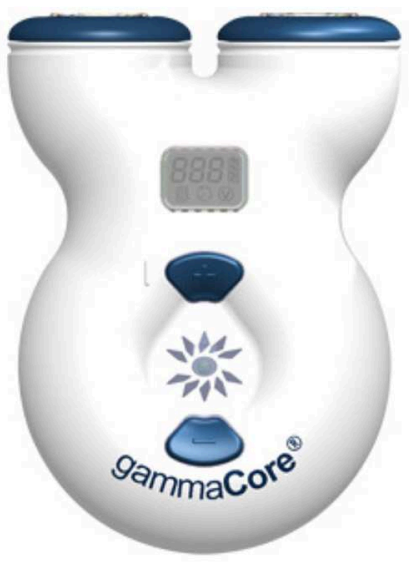

FIGURE 4 | (A) Cerbomed NEMOS. Adapted from www.cerbomed.com. (B). Electrocore gammaCore. Adapted from www.gammacore.com.

CE mark for the treatment of resistant epilepsy. It is comprised of two main components: the stimulation unit, which houses the battery and pulse generator (and is roughly the size of a mobile phone), and a dedicated ear electrode, which is connected to the stimulator via a cable. Stimulation intensity is user-controlled (up to $25 \mathrm{~V}$ ), with treatments lasting at least $1 \mathrm{~h}$ in three to four sessions per day for a total of 4-5 h. The stimulation current is adjusted until a slight tingling or pulsating sensation is perceived at the stimulation site, implying $A \beta$ fiber activation. Prior to stimulation, the user must clean the site of stimulation, as well as the electrodes, to minimize impedance and ensure optimal conductivity. The remaining stimulation parameters are fixed, delivering continuous 0.25 -ms-duration monophasic square wave pulses at $25 \mathrm{~Hz}$. Adverse effects may include a slight pain, burning, tingling or itching feeling under the electrode, which dissipates upon electrode removal.

\subsubsection{Other}

In addition to NEMOS and gammaCore, which are both manufactured specifically for tVNS, stimulation can also be performed by transcutaneous electrical nerve stimulator (TENS) devices, such as TENS-200, V-TENS PLUS, or TENS-NET 2000. Auri-Stim Medical have taken conventional TENS machines, which are typically used in pain management, and repurposed them for stimulating the ear by integrating the electrodes into a headset that can be worn by the user. These devices are portable battery powered control units that can administer tVNS in much the same way as the custom-built units, provided that the electrodes are placed in the correct location in the concha.

The TENS-NET 2000 was approved by the FDA in 2006 and labeled as a nerve stimulator for therapeutic use in depression, anxiety and depression (Hein et al., 2012). Userprogrammable stimulation parameters include frequency $(0.5-$ $100 \mathrm{~Hz}$ ), intensity (0-6 mA), and mode of stimulation (normal, burst or modulated). However, the polarity of the pulses cannot be varied and are typically monophasic rectangular waves. The stimulation can also be delivered in combination with music or different sounds to enhance the therapeutic effects.

For trials in a clinical or research-based setting, mainspowered medical stimulators, such as Digitimer DS7A or DS5 can be used. These allow complete personalization of stimulation parameters but sacrifice portability. These stimulators are isolated from the mains and can be connected to a computer via $\mathrm{BNC}$ cable to allow custom stimulation protocols to be delivered. The Digitimer DS7 is a general-purpose nerve or muscle stimulator for human stimulation and can output up to $100 \mathrm{~mA}$. The frequency and pulse widths of the waves, as well as the duty cycle, are typically programmed on a computer and delivered to the stimulator via BNC cable. There is also the option of alternating the polarity of the pulses, which allows both monophasic and biphasic stimulation pulses to be output.

\subsection{Electrode Types}

Several studies report using gammaCore or NEMOS devices but do not specify stimulation electrode types (e.g., Goadsby et al., 2014; Grazzi et al., 2014; Huang et al., 2014; Altavilla et al., 2015; Barbanti et al., 2015; Nesbitt et al., 2015; Straube et al., 2015). In these cases, we assume that stimulation electrodes provided with the device were not modified for the study, and we report manufacture specifications for the gammaCore/NEMOS electrodes in Table 2 (noted with an asterisk).

When reported, the most commonly used stimulation electrodes are made of titanium (for the ear) (Hasan et al., 2015; Sellaro et al., 2015a,b; Fischer et al., 2018; Jongkees et al., 2018) or stainless silver (for the neck) (Kinfe et al., 2015b; Gaul et al., 2016; Grazzi et al., 2016; Lerman et al., 2016; Silberstein et al., 2016a,b). Silver is also used as an electrode material for stimulation of ABVN (e.g., Laqua et al., 2014; Capone et al., 2015; Weise et al., 2015; Badran et al., 2018a; Keute et al., 2019). Information about stimulation electrodes is often somewhat 
insufficient: the material or size of the electrodes are often not specified (Stefan et al., 2012; Hyvärinen et al., 2015; Weise et al., 2015; Fang et al., 2016; Yakunina et al., 2018). This limits our collective understanding of the electrode-tissue interface and its interactions. However, the fact that the patient-specific pain threshold is often set as the stimulation current provides some control for variations in the electrode-tissue impedance.

\subsection{Stimulation Site}

Out of 61 studies included in Table 2, 13 use the neck as a stimulation location (Figure 5A) (see Gaul et al., 2016; Grazzi et al., 2016; Lerman et al., 2016; Silberstein et al., 2016a,b among others). Discrepancies exist between reported stimulation locations within the studies that stimulate ABVN (Figures 5B-F). This is true even when the same device is used; for example, Straube et al. (2015) and Frangos et al. (2015) both use the NEMOS device, yet report the concha and cymba concha as the location of stimulation, respectively. The stimulation location is often dictated by the geometry of an electrode, with clip electrodes typically attached to tragus or concha (Figures 5C,D) (Lehtimäki et al., 2013; Mei et al., 2014; Straube et al., 2015; Fang et al., 2016; Rong et al., 2016; Liu et al., 2018). Often the outer audio canal is reported as a site for stimulation, without further clarification for the location of an electrode (Hasan et al., 2015; Sellaro et al., 2015a,b; Steenbergen et al., 2015). Given that studies have been done in different participant groups with different clinical conditions and with different stimulation parameters, it is difficult to conclude an optimal stimulation site for any particular disorder.

Initial investigations in this direction have been undertaken in Napadow et al. (2012) and Kraus et al. (2013). Napadow et al. concluded that the concha is the best site for stimulation, while Kraus et al. proposed that the anterior wall of the ear canal is the best for efficacy and participant's convenience. Studies, such as these are progressing in the right direction, but a more systematic approach is required to investigate the effect of the electrode placement on the ABVN recruitment and corresponding neural activations.

Although research groups acknowledge that the ABVN innervates the tragus, concha, and cymba concha as per Peuker and Filler's anatomical studies (Peuker and Filler, 2002), most do not mention antihelix innervation. Selection of the stimulation site appears to be arbitrary, either predetermined by the device employed in the experiment or based on other previous studies without providing any evidence or explanation for the designated stimulation site.

\subsection{Stimulation Waveform}

Most studies employ monophasic rectangular waveforms often set by the specifications of the device used (Hein et al., 2012; Busch et al., 2013; Stavrakis et al., 2015; Badran et al., 2018a; Yakunina et al., 2018), while some others report using biphasic waveform stimulation (Stefan et al., 2012; Hyvärinen et al., 2015; Liu et al., 2018). Lerman et al. (2016) and Silberstein et al. (2016b) reported using sinusoidal wave bursts; however, it is not clear from these studies whether this waveform is more optimal to activate neural fibers. The use of devices that employ "proprietary" or "modified" waveforms, such as electroCore's gammaCore, further hinders insights into the effect of stimulation waveforms on key research outcomes.

\subsection{Stimulation Intensity}

The justifications mentioned above are also employed to motivate the choice of stimulation parameters. Some studies have credited (Kraus et al., 2007; Polak et al., 2009) as having defined the optimal stimulation parameters for tVNS. However, further investigation suggests that these studies only elucidate the optimal stimulus intensity to induce the greatest vagus sensory evoked potential (VSEP) amplitudes (Polak et al., 2009), and that tVNS causes hypo- and hyperactivations of brain regions of interest relating to a decrease in depressive symptoms (Kraus et al., 2007). As Polak et al. (2009) have stated, "we chose a stimulation intensity of $8 \mathrm{~mA}$ allowing detection of sufficient VSEP amplitudes without perception of pain," which reveals nothing about the effects observed post-synaptically in various structures of the brain.

They also acknowledge that VSEP amplitudes are directly correlated to stimulation intensity (i.e., stimulation intensities $>8 \mathrm{~mA}$ would elicit even greater VSEP amplitudes). Similarly, the studies of Kraus et al. (2007) showed no systematic effects of stimulation parameters on brain activation, although they did illustrate that tVNS does indeed elicit acute changes in brain regions that are related to a decrease in depressive symptoms similar to those caused by VNS. Therefore, neither of these studies can claim to have identified the optimal stimulation parameters of tVNS for the greatest decrease in depressive symptoms or seizure occurrence.

Furthermore, despite electrical current values being reported, the amount, or amplitude, of energy delivered to tissues is largely unknown given the substantial effect of electrode and tissue impedance and need for precise placement (e.g., a stated current of $8 \mathrm{~mA}$ presupposes that there is no impact of tissue impedance variation, and therefore voltage, and also neglects waveform shape, rise/fall-time, or any resultant residual charge). The stimulation current is often set according to the subject's sensitivity or just below pain threshold (Napadow et al., 2012; Frangos et al., 2015; Cha et al., 2016; Lerman et al., 2016; Fischer et al., 2018; Yakunina et al., 2018). Given the different stimulation tolerance of different participants, stimulation amplitudes vary over a wide range (from $0.5 \mathrm{~mA}$ in Jongkees et al., 2018 to $12 \mathrm{~mA}$ in Trevizol et al., 2016). Undoubtedly, the stimulation electrode electrochemistry also contributes to the maximum current that is tolerated by a participant.

\subsection{Stimulation Frequency}

With regard to stimulation frequency, the currently used range of $20-30 \mathrm{~Hz}$ has never been validated for its therapeutic effects (Laqua et al., 2014). Following studies showing that stimulation frequencies of $50 \mathrm{~Hz}$ and above can cause major and irreversible damage to the vagus nerve during VNS (Agnew and McCreery, 1990), stimulation frequencies between 20 and $30 \mathrm{~Hz}$ were arbitrarily selected in order to limit adverse events associated with direct stimulation of the carotid sheath and were subsequently approved by the FDA (Groves and Brown, 2005). 
A

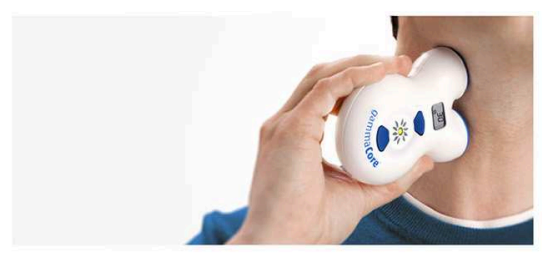

C

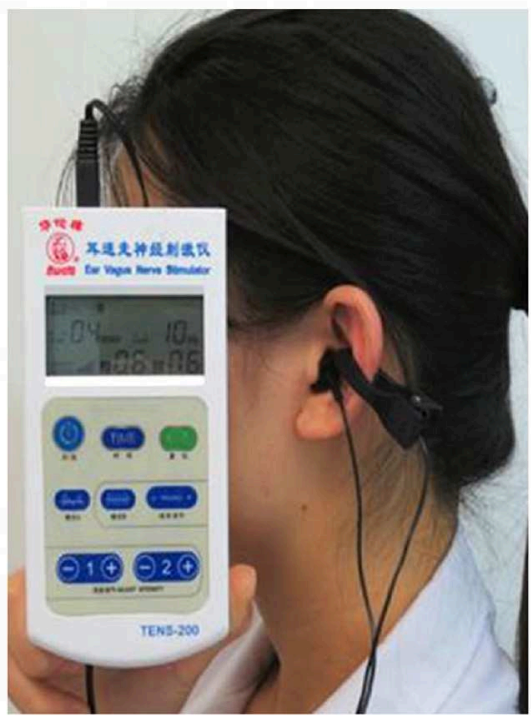

E

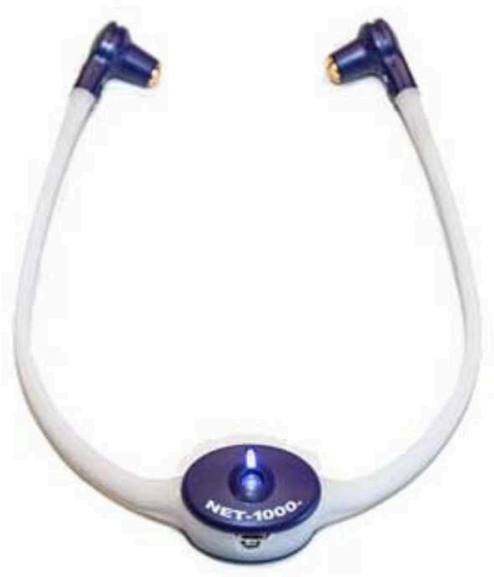

B

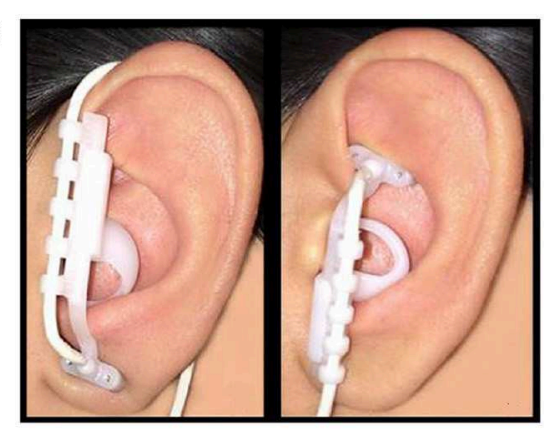

D

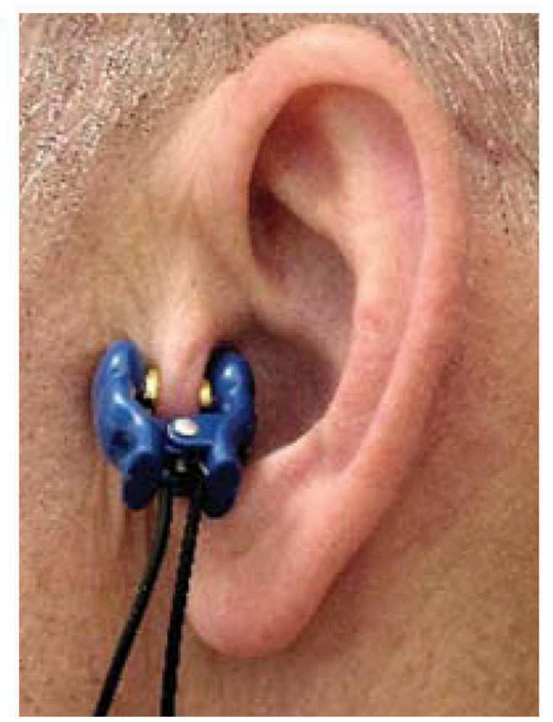

$\mathbf{F}$

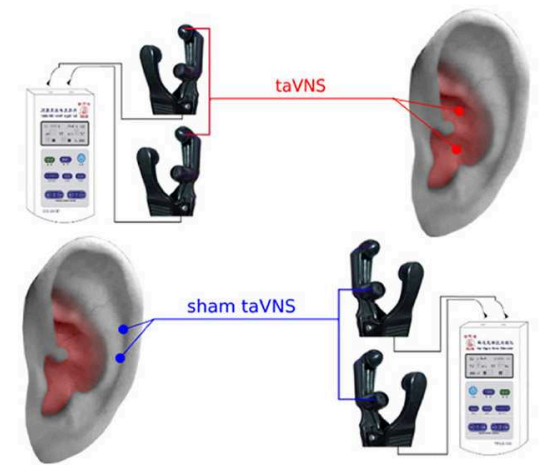

FIGURE 5 | Stimulation electrode positions. (A) Neck stimulation using a gammaCore device (Silberstein et al., 2016b). Image courtesy of electroCore Inc, electrocore.com. (B) Earlobe sham and cymba concha stimulation using NEMOS electrodes (Frangos et al., 2015). (C) External ear canal and concha stimulation using a TENS device from Suzhou (Liu et al., 2018). (D) Tragus stimulation (Lehtimäki et al., 2013). (E) External ear canal stimulation using a headset NET-1000 (Hein et al., 2012). Image courtesy of Auri-Stim Medical Inc, net1device.com. (F) Concha and cymba concha active stimulation (Rong et al., 2016). All figures reproduced with permission.

Lower frequencies of stimulation have also been explored. Liu et al. (2018) have found that $10 \mathrm{~Hz}$ tVNS for 20 min periods three times per day for 6 months reduced the number of seizures, while $8 \mathrm{~Hz}$ stimulation leads to activation in frontal and limbic brain areas as measured by fMRI (Kraus et al., 2007). Straube et al. (2015) have seen a stronger reduction in migraine episodes when stimulating at $1 \mathrm{~Hz}$ than when stimulating at $25 \mathrm{~Hz}$. Thus, it should not be assumed that stimulation frequencies within the 
20-30 Hz range are optimal for tVNS, and additional controlled studies are warranted to elucidate the effect of stimulation frequency rather than a selection based on past FDA approval of a related, yet different, technique.

\section{BRAIN ACTIVATION}

Several studies have speculated about the brain areas that are activated as a result of tVNS (Schulz-Stübner and Kehl, 2011; Busch et al., 2013; Laqua et al., 2014; Colzato et al., 2018; Jongkees et al., 2018). For example, Burger and Verkuil (2018) proposed that tVNS leads to activation in limbic areas, such as the amygdala and hippocampus, whereas Cha et al. (2016) suggested that it normalizes autonomic imbalance due to an increase in sympathetic response in patients with vertigo. In contrast, Silberstein et al. (2016b) proposed that stimulation of the vagus nerve affects hypocretin and orexin pathways in people with cluster headache, while Kinfe et al. (2015b) hypothesized that tVNS may help counteract the decline in thalamocortical activity in people with migraine and sleep disturbances. Jacobs et al. (2015) suggested that tVNS enhances memory performance by increasing neural activity in the locus coeruleus. It is clear that researchers have proposed different effects of tVNS on neural activation depending on the focus of their study. Measuring neural activity using techniques, such as fMRI, EEG, or MEG is critically important to confirm proposed hypotheses.

Brain activation in response to tVNS has been measured in Kraus et al. (2007), Kraus et al. (2013), Dietrich et al. (2008), Lehtimäki et al. (2013), Capone et al. (2015), Frangos et al. (2015), Hyvärinen et al. (2015), Weise et al. (2015), Fang et al. (2016), Yuan and Silberstein (2016b), Yu et al. (2017), Badran et al. (2018a), Fischer et al. (2018), Liu et al. (2018), Yakunina et al. (2018), Keute et al. (2019), Zhao et al. (2019), and Fallgatter et al. (2003). Most of these studies have been conducted in the last 5 years, with the exception of three that pioneered this field in the 2000s (Fallgatter et al., 2003; Kraus et al., 2007; Dietrich et al., 2008). Dietrich et al. (2008) showed that tVNS elicits activation in the left locus coeruleus, a brainstem nucleus that is implicated in clinical depression, as well as bilateral activation in the thalamus. Fallgatter et al. (2003) measured evoked potentials of post-synaptic brainstem activity from vagus nerve nuclei that can be elicited by electrical stimulation. Using fMRI, Kraus et al. (2007) demonstrated that tVNS leads to prominent changes in cerebral activation with marked deactivation in limbic and temporal brain areas.

Later fMRI studies have shown that active tVNS (i) produces a significantly larger increase in neural activity in the right caudate, bilateral anterior, left prefrontal cortex, cerebellum, and mid-cingulate than sham stimulation (Badran et al., 2018a); (ii) leads to a decrease in functional connectivity between posterior cingulate cortex and lingual gyrus (Zhao et al., 2019); and (iii) suppresses the auditory, limbic, and other brain areas implicated in the mechanisms involved in the generation of tinnitus (Yakunina et al., 2018).

EEG studies have shown a direct effect of tVNS on electrophysiological markers of conflict adaptation (Fischer et al.,
2018) and on the number of seizures (Liu et al., 2018). MEG recordings have shown that tVNS modulates synchrony of toneevoked brain activity, especially in the beta and gamma bands (Hyvärinen et al., 2015).

It is not clear why the areas of brain activation vary between these studies, but it may be due to the different conditions presented by the participants. Due to the variation in results, different studies have proposed different underlying mechanisms for tVNS, and, as such, there can be no clear conclusions made from the different imaging studies. Despite the breadth of research being undertaken, many questions remain regarding the most effective stimulation sites and parameters. As many of the described methods differ in the parameters and protocols applied, there is currently no firm evidence on the optimal parameters to provide the greatest benefit to subjects.

\subsection{Side Effects}

Although tVNS is on the whole well-tolerated as a treatment option, a number of different mild side effects have been noted, which Redgrave et al. (2018) summarized in their review. Common side effects include tingling or pain around the stimulation site, with some participants reporting itching or redness (Busch et al., 2013; He et al., 2013; Goadsby et al., 2014; Kreuzer et al., 2014; Rong et al., 2014; Barbanti et al., 2015; Hasan et al., 2015; Jacobs et al., 2015; Kinfe et al., 2015b; Stavrakis et al., 2015; Straube et al., 2015; Weise et al., 2015; Bauer et al., 2016; Cha et al., 2016; Grazzi et al., 2016; Lerman et al., 2016; Silberstein et al., 2016a,b; Trevizol et al., 2016). Other less common side effects that have been observed in $<1 \%$ of the study population include gastrointestinal issues, such as nausea or vomiting (Schulz-Stübner and Kehl, 2011; Kreuzer et al., 2014; Jacobs et al., 2015; Bauer et al., 2016; Silberstein et al., 2016b; Trevizol et al., 2016), headache (Stefan et al., 2012; Kreuzer et al., 2014; Jacobs et al., 2015; Bauer et al., 2016; Gaul et al., 2016; Lerman et al., 2016; Silberstein et al., 2016a; Trevizol et al., 2016), heart palpitations (Bauer et al., 2016), facial drooping (Goadsby et al., 2014; Silberstein et al., 2016b), dizziness (Aihua et al., 2014; Goadsby et al., 2014; Huang et al., 2014; Kreuzer et al., 2014; Rong et al., 2014; Jacobs et al., 2015; Bauer et al., 2016; Gaul et al., 2016), vocal hoarseness (Stefan et al., 2012; Goadsby et al., 2014; Kreuzer et al., 2014), and nasopharyingitis (Bauer et al., 2016; Gaul et al., 2016). There is currently no study that links stimulation parameters or dose to the rate of side effects experienced, which should be a priority for future research in the field, and clear reporting of both side effects and stimulation parameters is important to be able to observe any trends.

\section{DISCUSSION AND FUTURE DIRECTIONS}

This review has focused on a mechanistic understanding of transcutaneous vagus nerve stimulation (tVNS), with a detailed discussion of stimulation parameters, sites of stimulation, and devices used in current research. It should be noted that there 
is an ongoing discussion about the translation of non-invasive neural stimulation therapies into clinical practice. Transcranial magnetic stimulation (TMS) is another type of non-invasive neural stimulation therapy that is becoming more commonly used as a treatment option for different conditions, although use of the device is limited to clinical settings where it is operated by a healthcare professional. In contrast, transcranial direct stimulation (tDCS) (Wexler, 2015), much like tVNS, is a portable treatment option that does not require operation by a professional.

On the one hand, the affordability and easy availability of these devices, and an absence of severe adverse events, has led to a "do-it-yourself" movement that uses tDCS and tVNS at home for self-improvement purposes. Researchers are still trying to understand the risks and benefits of these techniques and fear that uncontrolled use may lead to unintended consequences (Bikson et al., 2013).

The situation is further complicated by the fact that, for regulatory purposes, the definition of a medical device focuses on the intended use of a device rather than the mechanism of action. This implies that manufacturers can skirt regulation by careful wording about the intended use. However, it is clear that a thorough risk analysis requires a sound understanding of the mechanism of action. Therefore, to promote the safe and efficacious use of tVNS in future, it is important to understand the mechanism of action of this promising technique.

The actual mechanisms of tVNS are still poorly understood. Many studies contradict the findings of similar studies and there is often very little homogeneity in results, making it difficult to draw conclusions from the findings. It has been proven by a number of studies that tVNS affects the same neural pathway as invasive VNS (He et al., 2009; Van Leusden et al., 2015); however, there is no conclusive evidence to explain why tVNS elicits therapeutic effects. It is therefore important for future studies to focus on the mechanism of action by following rigorous protocols that include objective measures of brain activation. It is also important that past assumptions about the effects of tVNS on brain neural activation and function do not restrict the direction of future investigations.

Given that stimulation parameters vary significantly between studies, a systematic approach is required to identify the optimal stimulation intensity, pulse width, waveform and frequency that provides the greatest clinical benefit. This may require participant-specific adjustment of parameters in a closed-loop setup, where stimulation parameters are set online, based on recorded neural activity. All current stimulation strategies for tVNS devices rely on open-loop control of the stimulation parameters, where the levels are set at the beginning of the stimulation protocol and do not change in response to any continuous measurement of the level of neuronal activation. It is reasonable to expect different outcomes in response to open-loop electrical stimulation between participants and between trials due to different ongoing brain activities at the time of stimulation. While many studies have been successful in using open-loop techniques (Barbanti et al., 2015; Trevizol et al., 2016; Liu et al., 2018), the outcomes differ from patient to patient. A customized closed-loop controller will allow the manipulation of specific patient-based neural responses. Pioneering steps in closed-loop VNS have been reported in Boon et al. (2015) and Fisher et al. (2016).

A closed-loop protocol will require continuous measurements of behavioral outcomes or brain activity. Since behavioral measures are often imprecise, it is preferable that imaging techniques, such as EEG or MEG, be used during the stimulation protocol to study neural activation and information transfer. The EEG signal has low spatial resolution that makes it difficult to interpret brain network connectivity. In contrast, MEG imaging has higher spatial resolution than EEG and higher temporal resolution than fMRI. The reconstruction of neuronal activity sources from MEG has less sensitivity to model approximations and smaller localization errors than EEG reconstruction. The MEG is sensitive to a wide range of frequencies in the oscillatory brain signals and has full brain coverage. There exist various techniques to reconstruct the anatomical origin of brain activity from MEG signal. When a structural MRI scan is available, it is possible to coregister MEG signals to anatomical locations. These advantages of MEG offer a powerful tool to study connectivity between brain areas and analyze brain networks and function (Baillet, 2017).

Such combined neuroimaging techniques can also help to resolve the origin of vagus connections in the brain. The "vagus" in the term tVNS is based on the assumption that the auricular branch of the vagus nerve has been activated. Some researchers believe that the auricular branch of the vagus is a misplaced branch of the trigeminal nerve and carries somatic-not visceralafferent fibers. In this respect, this nerve is just like the trigeminal nerve branches to the rest of the face. If this hypothesis is true, then the auricular nerve would not connect to the NTS in the brain but rather to the trigeminal-or possibly paratrigeminalnuclei. The latter nucleus receives cough receptor afferents from the airways, which may be why the auricular branch ("Arnold's nerve") can stimulate coughing (Gupta et al., 1986). However, a recent investigation of central neuronal projections from nerves innervating the external auricle in rats, appears to challenge an opinion that stimulation of the tragal nerve is conducted by the auricular branch of the vagus (Mahadi et al., 2019). Similar studies need to be done in primates to confirm whether the same conclusion may apply to humans.

Many studies have very few participants, with some having as few as one. This leads to difficulties in concluding whether the results or proposed mechanisms can be generalized to a larger population. To avoid the risk of accidentally having extreme or biased results, studies with a large number of participants are required. Due to heterogeneous populations with various health conditions and different medications and treatment responses often enlisted for a study, it is impossible to generalize to another condition or to a healthy group. Rigorous studies with a large number of healthy participants, where a wide range of stimulation parameters are tested within a participant and between a cohort, are needed to draw solid, evidencebased conclusions. Such studies may also reveal biomarkers for responders and non-responders to tVNS.

There has been very little investigation into how long the effects of tVNS last after the stimulation period has ended. Most 
clinical trials involve daily stimulation periods over the course of the trial, with the therapeutic results measured concurrently. Studies, such as that of Hein et al. (2012), have compared therapeutic results after the 2 -weeks treatment period of daily stimulation to the baseline results recorded from before the stimulation period. Other studies (Huang et al., 2014; Mei et al., 2014; Rong et al., 2016) measured the therapeutic effects of daily stimulation continuously over set intervals during the trial period. Many studies found that participants who completed the entire treatment study had a better response to tVNS than those who dropped out, and longer treatment periods corresponded with better therapeutic outcomes (He et al., 2013; Bauer et al., 2016; Silberstein et al., 2016a; Liu et al., 2018). However, these studies did not offer a follow-up to see whether the effects of tVNS were long-lasting or remained after the cessation of the treatment period. In the case study by Zhao et al. (2019) on a single participant with insomnia, after 2 weeks of twice daily tVNS the treatment was stopped, but the participant still felt an improvement at the follow-up meeting, 3 months after the trial period. Similarly, Trevizol et al. (2016) had a stimulation period of 10 days, but found the clinical response remained stable 1 month after stimulation had stopped.

Some studies into the pain-relieving effects of tVNS have investigated whether the effects last for some time after the stimulation. Johnson et al. (1991) and Napadow et al. (2012) reported that an analgesic effect was present for up to $15 \mathrm{~min}$ after stimulation ceased. Other studies measured the therapeutic effects immediately after stimulation (Capone et al., 2015; Stavrakis et al., 2015; Keute et al., 2019) or at the same time as stimulation (Fallgatter et al., 2003; Kraus et al., 2007, 2013; Dietrich et al., 2008; Lehtimäki et al., 2013). This may offer interesting results for the measurement of brain activity as a result of tVNS but does not indicate whether these effects are long-lasting. Indeed, Frangos et al. (2015) noted that neural activation gradually returned to the baseline after tVNS was stopped. Immediate measurement of the therapeutic effects of

\section{REFERENCES}

Agnew, W. F., and McCreery, D. B. (1990). Considerations for safety with chronically implanted nerve electrodes. Epilepsia 31, S27-S32. doi: 10.1111/j.1528-1157.1990.tb05845.x

Aihua, L., Lu, S., Liping, L., Xiuru, W., Hua, L., and Yuping, W. (2014). A controlled trial of transcutaneous vagus nerve stimulation for the treatment of pharmacoresistant epilepsy. Epilepsy Behav. 39, 105-110. doi: 10.1016/j.yebeh.2014.08.005

Altavilla, R., Paolucci, M., Altamura, C., and Vernieri, F. (2015). P038. Effects of non-invasive vagus nerve stimulation on cerebral vasomotor reactivity in patients with chronic migraine during intercritical phase: a pilot study. J. Headache Pain 16:A62. doi: 10.1186/1129-2377-16-S1-A62

Ardell, J. L., and Randall, W. C. (1986). Selective vagal innervation of sinoatrial and atrioventricular nodes in canine heart. Am. J. Physiol. Heart Circ. Physiol. 251, H764-H773. doi: 10.1152/ajpheart.1986.251.4.H764

Assenza, G., Campana, C., Colicchio, G., Tombini, M., Assenza, F., Di Pino, G., et al. (2017). Transcutaneous and invasive vagal nerve stimulations engage the same neural pathways: in-vivo human evidence. Brain Stimul. 10, 853-854. doi: 10.1016/j.brs.2017.03.005
tVNS do not therefore suggest whether these effects are merely a temporary result of stimulation or long-lasting.

When long periods of stimulation are required to achieve the maximum effect, it is unreasonable to expect participants to attend prolonged sessions several times per day. Therefore, portable stimulators are required, but gammaCore and NEMOS are currently the only tVNS devices available. It is difficult to track participants' compliance with these devices and record how stimulation parameters change over time. More research is required to produce miniaturized devices that are convenient and safe to use.

\section{CONCLUSION}

tVNS has proven to be an effective way to modulate the central nervous system in some cases. However, the mechanism of action is not clear, and the robustness of the results is yet to be proven. The technique is safe and convenient with only a few relatively minor side effects reported. More rigorous systematic studies are required to investigate the effects of stimulation parameters, sites of stimulation, and electrode types on brain activation and clinical outcomes. Current limitations in study protocols may lead to difficulties in obtaining regulatory approval and challenges in translating research studies into clinical practice.

\section{AUTHOR CONTRIBUTIONS}

JY and PS conceived and designed the idea. JY, CK, TK, and PS wrote the manuscript. All authors aided in interpreting the results and commented on the manuscript.

\section{FUNDING}

PS and JY acknowledge funding support from the Australian Research Council (IC140100023) and Grey Innovation Pty Ltd.

Badran, B. W., Dowdle, L. T., Mithoefer, O. J., LaBate, N. T., Coatsworth, J., Brown, J. C., et al. (2018a). Neurophysiologic effects of transcutaneous auricular vagus nerve stimulation (taVNS) via electrical stimulation of the tragus: a concurrent taVNS/fMRI study and review. Brain Stimul. 11, 492-500. doi: 10.1016/j.brs.2017.12.009

Badran, B. W., Jenkins, D. D., DeVries, W. H., Dancy, M., Summers, P. M., Mappin, G. M., et al. (2018b). Transcutaneous auricular vagus nerve stimulation (taVNS) for improving oromotor function in newborns. Brain Stimul. 11, 1198-1200. doi: 10.1016/j.brs.2018.06.009

Baillet, S. (2017). Magnetoencephalography for brain electrophysiology and imaging. Nat. Neurosci. 20, 327-339. doi: 10.1038/nn.4504

Barbanti, P., Grazzi, L., Egeo, G., Maria Padovan, A., Liebler, E., and Bussone, G. (2015). Non-invasive vagus nerve stimulation for acute treatment of highfrequency and chronic migraine: an open-label study. J. Headache Pain 16:61. doi: 10.1186/s10194-015-0542-4

Bauer, S., Baier, H., Baumgartner, C., Bohlmann, K., Fauser, S., Graf, W., et al. (2016). Transcutaneous vagus nerve stimulation (tVNS) for treatment of drug-resistant epilepsy: a randomized, double-blind clinical trial (cMPsE02). Brain Stimul. 9, 356-363. doi: 10.1016/j.brs.2015. 11.003 
Beghi, E. (2019). Global, regional, and national burden of epilepsy, 1990-2016: a systematic analysis for the Global Burden of Disease Study 2016. Lancet Neurol. 18, 357-375. doi: 10.1016/S1474-4422(18)30454-X

Ben-Menachem, E., Hamberger, A., Hedner, T., Hammond, E., Uthman, B., Slater, J., et al. (1995). Effects of vagus nerve stimulation on amino acids and other metabolites in the CSF of patients with partial seizures. Epilepsy Res. 20, 221-227. doi: 10.1016/0920-1211(94)00083-9

Bikson, M., Bestmann, S., and Edwards, D. (2013). Transcranial devices are not playthings. Nature 501, 167-167. doi: 10.1038/501167b

Boon, P., Vonck, K., van Rijckevorsel, K., Tahry, R. E., Elger, C. E., Mullatti, N., et al. (2015). A prospective, multicenter study of cardiac-based seizure detection to activate vagus nerve stimulation. Seizure 32, 52-61. doi: $10.1016 /$ j.seizure.2015.08.011

Burger, A. M., and Verkuil, B. (2018). Transcutaneous nerve stimulation via the tragus: are we really stimulating the vagus nerve? Brain Stimul. 11, 945-946. doi: 10.1016/j.brs.2018.03.018

Burger, A. M., Verkuil, B., Van Diest, I., Van der Does, W., Thayer, J. F., and Brosschot, J. F. (2016). The effects of transcutaneous vagus nerve stimulation on conditioned fear extinction in humans. Neurobiol. Learn. Mem. 132, 49-56. doi: 10.1016/j.nlm.2016.05.007

Busch, V., Zeman, F., Heckel, A., Menne, F., Ellrich, J., and Eichhammer, P. (2013). The effect of transcutaneous vagus nerve stimulation on pain perception-an experimental study. Brain Stimul. 6, 202-209. doi: 10.1016/j.brs.2012.04.006

Butt, M. F., Albusoda, A., Farmer, A. D., and Aziz, Q. (2019). The anatomical basis for transcutaneous auricular vagus nerve stimulation. J. Anat. 236, 588-611. doi: $10.1111 /$ joa.13122

Capone, F., Assenza, G., Di Pino, G., Musumeci, G., Ranieri, F., Florio, L., et al. (2015). The effect of transcutaneous vagus nerve stimulation on cortical excitability. J. Neural Transm. 122, 679-685. doi: 10.1007/s00702-014$1299-7$

Cha, W. W., Song, K., and Lee, H. Y. (2016). Persistent geotropic directionchanging positional nystagmus treated with transcutaneous vagus nerve stimulation. Brain Stimul. 9, 469-470. doi: 10.1016/j.brs.2016. 03.011

Chae, J. H., Nahas, Z., Lomarev, M., Denslow, S., Lorberbaum, J. P., Bohning, D. E., et al. (2003). A review of functional neuroimaging studies of vagus nerve stimulation (VNS). J. Psychiatr. Res. 37, 443-455. doi: 10.1016/S0022-3956(03)00074-8

Chen, M., Yu, L., Ouyang, F., Liu, Q., Wang, Z., Wang, S., et al. (2015). The right side or left side of noninvasive transcutaneous vagus nerve stimulation: based on conventional wisdom or scientific evidence? Int. J. Cardiol. 187, 44-45. doi: 10.1016/j.ijcard.2015.03.351

Clancy, J. A., Mary, D. A., Witte, K. K., Greenwood, J. P., Deuchars, S. A., and Deuchars, J. (2014). Non-invasive vagus nerve stimulation in healthy humans reduces sympathetic nerve activity. Brain Stimul. 7, 871-877. doi: 10.1016/j.brs.2014.07.031

Colzato, L. S., Ritter, S. M., and Steenbergen, L. (2018). Transcutaneous vagus nerve stimulation (tVNS) enhances divergent thinking. Neuropsychologia 111, 72-76. doi: 10.1016/j.neuropsychologia.2018.01.003

Coppola, G., Vandenheede, M., Di Clemente, L., Ambrosini, A., Fumal, A., De Pasqua, V., et al. (2004). Somatosensory evoked high-frequency oscillations reflecting thalamo-cortical activity are decreased in migraine patients between attacks. Brain 128, 98-103. doi: 10.1093/brain/awh334

Depression Physician's Manual (2005). Depression Physician's Manual: VNS Therapy Pulse Model 102 Generator and VNS Therapy Pulse Duo Model 102R Generator.

Dietrich, S., Smith, J., Scherzinger, C., Hofmann-Preiß, K., Freitag, T., Eisenkolb, A., et al. (2008). A novel transcutaneous vagus nerve stimulation leads to brainstem and cerebral activations measured by functional MRI [Funktionelle Magnetresonanztomographie zeigt Aktivierungen des Hirnstamms und weiterer zerebraler Strukturen unter transkutaner Vagusne]. Biomed. Eng. 53, 104-111. doi: 10.1515/BMT.2008.022

Dorr, A. E., and Debonnel, G. (2006). Effect of vagus nerve stimulation on serotonergic and noradrenergic transmission. J. Pharmacol. Exp. Ther. 318, 890-898. doi: 10.1124/jpet.106.104166

ElectroCore, I. (2018). Instructions for Use for gammaCore Sapphire ${ }^{T M}$ (Noninvasive Vagus Nerve Stimulator). Technical report, tVNS device GammaCore, electroCore Inc.
Engineer, N. D., Riley, J. R., Seale, J. D., Vrana, W. A., Shetake, J. A., Sudanagunta, S. P., et al. (2011). Reversing pathological neural activity using targeted plasticity. Nature 470, 101-104. doi: 10.1038/nature09656

Evans, M. S., Verma-Ahuja, S., Naritoku, D. K., and Espinosa, J. A. (2004). Intraoperative human vagus nerve compound action potentials. Acta Neurol. Scand. 110, 232-238. doi: 10.1111/j.1600-0404.2004.00309.x

Fahy, B. G. (2010). Intraoperative and perioperative complications with a vagus nerve stimulation device. J. Clin. Anesth. 22, 213-222. doi: 10.1016/j.jclinane.2009.10.002

Fallgatter, A. J., Neuhauser, B., Herrmann, M. J., Ehlis, A.-C., Wagener, A., Scheuerpflug, P., et al. (2003). Far field potentials from the brain stem after transcutaneous vagus nerve stimulation. J. Neural Transm. 110, 1437-1443. doi: 10.1007/s00702-003-0087-6

Fang, J., Egorova, N., and Rong, P. (2017). Early cortical biomarkers of longitudinal transcutaneous vagus nerve stimulation treatment success in depression. Neuroimage Clin. 14, 105-111. doi: 10.1016/j.nicl.2016.12.016

Fang, J., Rong, P., Hong, Y., Fan, Y., Liu, J., Wang, H., et al. (2016). Transcutaneous vagus nerve stimulation modulates default mode network in major depressive disorder. Biol. Psychiatry 79, 266-273. doi: 10.1016/j.biopsych.2015. 03.025

Fischer, R., Ventura-Bort, C., Hamm, A., and Weymar, M. (2018). Transcutaneous vagus nerve stimulation (tVNS) enhances conflict-triggered adjustment of cognitive control. Cogn. Affect. Behav. Neurosci. 18, 680-693. doi: 10.3758/s13415-018-0596-2

Fisher, R. S., Afra, P., Macken, M., Minecan, D. N., Bagić, A., Benbadis, S. R., et al. (2016). Automatic vagus nerve stimulation triggered by ictal tachycardia: clinical outcomes and device performance-the U.S. E-37 trial. Neuromodulation 19, 188-195. doi: 10.1111/ner.12376

Fitzgerald, P. B., Jessica Benitez, F., Anthony de Castella, G., Jeff Daskalakis, D. Z., and Kulkarni, J. (2006). A Randomized, Controlled Trial of Sequential Bilateral Repetitive Transcranial Magnetic Stimulation for Treatment-Resistant Depression. Technical report.

Fix, J. D., and Brueckner, J. K. (2009). High-Yield Neuroanatomy. Philadelphia, PA: Wolters Kluwer Health; Lippincott Williams \& Wilkins.

Frangos, E., Ellrich, J., and Komisaruk, B. R. (2015). Non-invasive access to the vagus nerve central projections via electrical stimulation of the external ear: fMRI evidence in humans. Brain Stimul. 8, 624-636. doi: 10.1016/j.brs.2014.11.018

Frokaer, J. B., Bergmann, S., Brock, C., Madzak, A., Farmer, A. D., Ellrich, J., et al. (2016). Modulation of vagal tone enhances gastroduodenal motility and reduces somatic pain sensitivity. Neurogastroenterol. Motil. 28, 592-598. doi: $10.1111 / \mathrm{nmo} .12760$

Gaul, C., Diener, H.-C., Silver, N., Magis, D., Reuter, U., Andersson, A., et al. (2016). Non-invasive vagus nerve stimulation for PREVention and Acute treatment of chronic cluster headache (PREVA): a randomised controlled study. Cephalalgia 36, 534-546. doi: 10.1177/0333102415607070

Goadsby, P., Grosberg, B., Mauskop, A., Cady, R., and Simmons, K. (2014). Effect of noninvasive vagus nerve stimulation on acute migraine: an open-label pilot study. Cephalalgia 34, 986-993. doi: 10.1177/0333102414524494

Grazzi, L., Egeo, G., Calhoun, A. H., McClure, C. K., Liebler, E., and Barbanti, P. (2016). Non-invasive vagus nerve stimulation (nVNS) as mini-prophylaxis for menstrual/menstrually related migraine: an open-label study. J. Headache Pain 17:91. doi: 10.1186/s10194-016-0684-z

Grazzi, L., Usai, S., and Bussone, G. (2014). Gammacore device for treatment of migraine attack: preliminary report. J. Headache Pain 15:G12. doi: 10.1186/1129-2377-15-S1-G12

Groves, D. A., and Brown, V. J. (2005). Vagal nerve stimulation: a review of its applications and potential mechanisms that mediate its clinical effects. Neurosci. Biobehav. Rev. 29, 493-500. doi: 10.1016/j.neubiorev.2005.01.004

Gupta, D., Verma, S., and Vishwakarma, S. (1986). Anatomic basis of Arnold's ear-cough reflex. Surg. Radiol. Anat. 8, 217-220. doi: 10.1007/BF02425070

Hammer, N., Löffler, S., Cakmak, Y. O., Ondruschka, B., Planitzer, U., Schultz, M., et al. (2018). Cervical vagus nerve morphometry and vascularity in the context of nerve stimulation-a cadaveric study. Nat. Sci. Rep. 8:7997. doi: 10.1038/s41598-018-26135-8

Han, B. I., Lee, H. W., Kim, T. Y., Lim, J. S., and Shin, K. S. (2009). Tinnitus: characteristics, causes, mechanisms, and treatments. J. Clin. Neurol. 5:11. doi: 10.3988/jen.2009.5.1.11 
Handforth, A., DeGiorgio, C. M., Schachter, S. C., Uthman, B. M., Naritoku, D. K., Tecoma, E. S., et al. (1998). Vagus nerve stimulation therapy for partial-onset seizures: a randomized active-control trial. Neurology 51, 48-55. doi: 10.1212/WNL.51.1.48

Hasan, A., Wolff-Menzler, C., Pfeiffer, S., Falkai, P., Weidinger, E., Jobst, A., et al. (2015). Transcutaneous noninvasive vagus nerve stimulation (tVNS) in the treatment of schizophrenia: a bicentric randomized controlled pilot study. Eur. Archiv. Psychiatry Clin. Neurosci. 265, 589-600. doi: 10.1007/s00406-015-0618-9

He, W., Jing, X., Wang, X., Rong, P., Li, L., Shi, H., et al. (2013). Transcutaneous auricular vagus nerve stimulation as a complementary therapy for pediatric epilepsy: a pilot trial. Epilepsy Behav. 28, 343-346. doi: 10.1016/j.yebeh.2013.02.001

He, W., Wang, X., Shi, H., Shang, H., Li, L., Jing, X., et al. (2012). Auricular acupuncture and vagal regulation. Evid. Based Complement. Altern. Med. 2012, 1-6. doi: 10.1155/2012/615476

He, W., Zhu, B., and Rong, P. (2009). A new concept of transcutaneous vagus nerve stimulation for epileptic seizure. Abstr. Soc. Neurosci. 539. doi: 10.1155/2012/786839

Hein, E., Nowak, M., Kiess, O., Biermann, T., Bayerlein, K., Kornhuber, J., et al. (2012). Auricular transcutaneous electrical nerve stimulation in depressed patients: a randomized controlled pilot study. J. Neural Transm. 120, 821-827. doi: 10.1007/s00702-012-0908-6

Helmers, S. L., Begnaud, J., Cowley, A., Corwin, H. M., Edwards, J. C., Holder, D. L., et al. (2012). Application of a computational model of vagus nerve stimulation. Acta Neurol. Scand. 126, 336-343. doi: 10.1111/j.1600-0404.2012.01656.x

Henry, T. R. (2002). Therapeutic mechanisms of vagus nerve stimulation. Neurology 59, S3-S14. doi: 10.1212/WNL.59.6_suppl_4.S3

Henry, T. R., Bakay, R. A. E., Votaw, J. R., Pennell, P. B., Epstein, C. M., Faber, T. L., et al. (1998). Brain blood flow alterations induced by therapeutic vagus nerve stimulation in partial epilepsy: I. Acute effects at high and low levels of stimulation. Epilepsia 39, 983-990. doi: 10.1111/j.1528-1157.1998.tb 01448.x

Huang, F., Dong, J., Kong, J., Wang, H., Meng, H., Spaeth, R. B., et al. (2014). Effect of transcutaneous auricular vagus nerve stimulation on impaired glucose tolerance: a pilot randomized study. BMC Complement. Altern. Med. 14:203. doi: 10.1186/1472-6882-14-203

Hyvärinen, P., Yrttiaho, S., Lehtimäki, J., Ilmoniemi, R. J., Mäkitie, A., Ylikoski, J., et al. (2015). Transcutaneous vagus nerve stimulation modulates tinnitus-related beta- and gamma-band activity. Ear Hear. 36, e76-e85. doi: 10.1097/AUD.0000000000000123

Iriarte, J., Urrestarazu, E., Alegre, M., Macías, A., Gómez, A., Amaro, P., et al. (2009). Late-onset periodic asystolia during vagus nerve stimulation. Epilepsia 50, 928-932. doi: 10.1111/j.1528-1167.2008.01918.x

Jacobs, H. I., Riphagen, J. M., Razat, C. M., Wiese, S., and Sack, A. T. (2015). Transcutaneous vagus nerve stimulation boosts associative memory in older individuals. Neurobiol. Aging 36, 1860-1867. doi: 10.1016/j.neurobiolaging.2015.02.023

Johnson, M., Hajela, V., Ashton, C., and Thompson, J. (1991). The effects of auricular transcutaneous electrical nerve stimulation (TENS) on experimental pain threshold and autonomic function in healthy subjects. Pain 46, 337-342. doi: 10.1016/0304-3959(91)90116-F

Johnson, R. L., and Wilson, C. G. (2018). A review of vagus nerve stimulation as a therapeutic intervention. J. Inflamm. Res. 11, 203-213. doi: $10.2147 /$ JIR.S163248

Jongkees, B. J., Immink, M. A., Finisguerra, A., and Colzato, L. S. (2018). Transcutaneous vagus nerve stimulation (tVNS) enhances response selection during sequential action. Front. Psychol. 9:1159. doi: 10.3389/fpsyg.2018.01159

Kaniusas, E., Kampusch, S., Tittgemeyer, M., Panetsos, F., Gines, R. F., Papa, M., et al. (2019a). Current directions in the auricular vagus nerve stimulation II-an engineering perspective. Front. Neurosci. 13:772. doi: 10.3389/fnins.2019.00772

Kaniusas, E., Kampusch, S., Tittgemeyer, M., Panetsos, F., Gines, R. F., Papa, M., et al. (2019b). Current directions in the auricular vagus nerve stimulation I-a physiological perspective. Front. Neurosci. 13:854. doi: 10.3389/fnins.2019.00854

Keute, M., Boehrer, L., Ruhnau, P., Heinze, H.-J., and Zaehle, T. (2019). Transcutaneous vagus nerve stimulation (tVNS) and the dynamics of visual bistable perception. Front. Neurosci. 13:227. doi: 10.3389/fnins.2019.00227
Keute, M., Ruhnau, P., Heinze, H. J., and Zaehle, T. (2018). Behavioral and electrophysiological evidence for GABAergic modulation through transcutaneous vagus nerve stimulation. Clin. Neurophysiol. 129, 1789-1795. doi: 10.1016/j.clinph.2018.05.026

Kinfe, T. M., Pintea, B., Güresir, E., and Vatter, H. (2015a). Partial response of intractable cluster-Tic syndrome treated by cervical non-invasive vagal nerve stimulation (nVNS). Brain Stimul. 8, 669-671. doi: 10.1016/j.brs.2015.01.002

Kinfe, T. M., Pintea, B., Muhammad, S., Zaremba, S., Roeske, S., Simon, B. J., et al. (2015b). Cervical non-invasive vagus nerve stimulation (nVNS) for preventive and acute treatment of episodic and chronic migraine and migraine-associated sleep disturbance: preliminary findings from a prospective observational cohort study. J. Headache Pain 16:101. doi: 10.1186/s10194-015-0582-9

Kiyokawa, J., Yamaguchi, K., Okada, R., Maehara, T., and Akita, K. (2014). Origin, course and distribution of the nerves to the posterosuperior wall of the external acoustic meatus. Anat. Sci. Int. 89, 238-245. doi: 10.1007/s12565-014-0231-4

Krahl, S. (2012). Vagus nerve stimulation for epilepsy: a review of the peripheral mechanisms. Surg. Neurol. Int. 3:47. doi: 10.4103/2152-7806.103015

Krahl, S. E., Clark, K. B., Smith, D. C., and Browning, R. A. (1998). Locus coeruleus lesions suppress the seizure-attenuating effects of vagus nerve stimulation. Epilepsia 39, 709-714. doi: 10.1111/j.1528-1157.1998.tb01155.x

Kraus, T., Hösl, K., Kiess, O., Schanze, A., Kornhuber, J., and Forster, C. (2007). BOLD fMRI deactivation of limbic and temporal brain structures and mood enhancing effect by transcutaneous vagus nerve stimulation. J. Neural Transm. 114, 1485-1493. doi: 10.1007/s00702-007-0755-Z

Kraus, T., Kiess, O., Hösl, K., Terekhin, P., Kornhuber, J., and Forster, C. (2013). CNS BOLD fMRI effects of sham-controlled transcutaneous electrical nerve stimulation in the left outer auditory canal-a pilot study. Brain Stimul. 6, 798-804. doi: 10.1016/j.brs.2013.01.011

Kreuzer, P. M., Landgrebe, M., Resch, M., Husser, O., Schecklmann, M., Geisreiter, F., et al. (2014). Feasibility, safety and efficacy of transcutaneous vagus nerve stimulation in chronic tinnitus: an open pilot study. Brain Stimul. 7, 740-747. doi: 10.1016/j.brs.2014.05.003

Kwan, P., and Brodie, M. J. (2000). Early identification of refractory epilepsy. N. Engl. J. Med. 342, 314-319. doi: 10.1056/NEJM200002033420503

Labiner, D. M., and Ahern, G. L. (2007). Vagus nerve stimulation therapy in depression and epilepsy: therapeutic parameter settings. Acta Neurol. Scand. 115, 23-33. doi: 10.1111/j.1600-0404.2006.0 0732.x

Laqua, R., Leutzow, B., Wendt, M., and Usichenko, T. (2014). Transcutaneous vagal nerve stimulation may elicit anti- and pro-nociceptive effects under experimentally-induced pain-a crossover placebo-controlled investigation. Auton. Neurosci. Basic Clin. 185, 120-122. doi: 10.1016/j.autneu.2014. 07.008

Lehtimäki, J., Hyvärinen, P., Ylikoski, M., Bergholm, M., Mäkelä, J. P., Aarnisalo, A., et al. (2013). Transcutaneous vagus nerve stimulation in tinnitus: a pilot study. Acta Oto-Laryngol. 133, 378-382. doi: 10.3109/00016489.2012.75 0736

Lerman, I., Hauger, R., Sorkin, L., Proudfoot, J., Davis, B., Huang, A., et al. (2016). Noninvasive Transcutaneous vagus nerve stimulation decreases whole blood culture-derived cytokines and chemokines: a randomized, blinded, healthy control pilot trial. Neuromodulation 19, 283-290. doi: 10.1111/ner. 12398

Liu, A., Rong, P., Gong, L., Song, L., Wang, X., Li, L., et al. (2018). Efficacy and safety of treatment with transcutaneous vagus nerve stimulation in 17 patients with refractory epilepsy evaluated by electroencephalogram, seizure frequency, and quality of life. Med. Sci. Monit. 24, 8439-8448. doi: 10.12659/MSM.9 10689

Mahadi, K., Lall, V., Deuchars, S., and Deuchars, J. (2019). Cardiovascular autonomic effects of transcutaneous auricular nerve stimulation via the tragus in the rat involve spinal cervical sensory afferent pathways. Brain Stimul. 12, 1151-1158. doi: 10.1016/j.brs.2019.05.002

Manta, S., Dong, J., Debonnel, G., and Blier, P. (2009). Enhancement of the function of rat serotonin and norepinephrine neurons by sustained vagus nerve stimulation. J. Psychiatry Neurosci. 34, 272-280.

Marzec, M., Edwards, J., Sagher, O., Fromes, G., and Malow, B. A. (2003). Effects of vagus nerve stimulation on sleep-related breathing in epilepsy patients. Epilepsia 44, 930-935. doi: 10.1046/j.1528-1157.2003.5 6202.x 
Mayberg, H. S. (1997). Limbic-cortical dysregulation: a proposed model of depression. J. Neuropsychiatr. Clin. Neurosci. 9, 471-481. doi: $10.1176 /$ jnp.9.3.471

Mayberg, H. S., Brannan, S. K., Tekell, J. L., Silva, J. A., Mahurin, R. K., McGinnis, S., et al. (2000). Regional metabolic effects of fluoxetine in major depression: serial changes and relationship to clinical response. Biol. Psychiatry 48, 830-843. doi: 10.1016/S0006-3223(00)01036-2

Mei, Z., Yang, S., Cai, S., Lei, H., Zhou, C., Guo, Y., et al. (2014). Treatment of tinnitus with electrical stimulation on acupoint in the distribution area of ear vagus nerve combining with sound masking: randomized controlled trial. World J. Acupunct. Moxibust. 24, 30-35. doi: 10.1016/S1003-5257(14)60022-2

Mertens, A., Raedt, R., Gadeyne, S., Carrette, E., Boon, P., and Vonck, K. (2018). Recent advances in devices for vagus nerve stimulation. Expert Rev. Med. Devices 15, 527-539. doi: 10.1080/17434440.2018.15 07732

Mohr, P., Rodriguez, M., Slavíčková, A., and Hanka, J. (2011). The application of vagus nerve stimulation and deep brain stimulation in depression. Neuropsychobiology 64, 170-181. doi: 10.1159/0003 25225

Mourdoukoutas, A. P., Truong, D. Q., Adair, D. K., Simon, B. J., and Bikson, M. (2018). High-resolution multi-scale computational model for noninvasive cervical vagus nerve stimulation. Neuromodulation 21, 261-268. doi: 10.1111/ner.12706

Müller, H. H. O., Moeller, S., Lücke, C., Lam, A. P., Braun, N., and Philipsen, A. (2018). Vagus nerve stimulation (VNS) and other augmentation strategies for therapy-resistant depression (TRD): review of the evidence and clinical advice for use. Front. Neurosci. 12:239. doi: 10.3389/fnins.2018. 00239

Multon, S., and Schoenen, J. (2005). Pain control by vagus nerve stimulation: from animal to man...and back. Acta Neurol. Belg. 105, 62-67.

Napadow, V., Edwards, R. R., Cahalan, C. M., Mensing, G., Greenbaum, S., Valovska, A., et al. (2012). Evoked pain analgesia in chronic pelvic pain patients using respiratory-gated auricular vagal afferent nerve stimulation. Pain Med. 13, 777-789. doi: 10.1111/j.1526-4637.2012. 01385.x

Nemeroff, C. B., Mayberg, H. S., Krahl, S. E., McNamara, J., Frazer, A., Henry, T. R., et al. (2006). VNS therapy in treatment-resistant depression: clinical evidence and putative neurobiological mechanisms. Neuropsychopharmacology 31, 1345-1355. doi: 10.1038/sj.npp. 1301190

Nesbitt, A. D., Marin, J. C. A., Tompkins, E., Ruttledge, M. H., and Goadsby, P. J. (2015). Initial use of a novel noninvasive vagus nerve stimulator for cluster headache treatment. Neurology 84, 1249-1253. doi: 10.1212/WNL.0000000000001394

Peuker, E. T., and Filler, T. J. (2002). The nerve supply of the human auricle. Clin. Anat. 15, 35-37. doi: 10.1002/ca.1089

Polak, T., Markulin, F., Ehlis, A.-C., Langer, J. B. M., Ringel, T. M., and Fallgatter, A. J. (2009). Far field potentials from brain stem after transcutaneous vagus nerve stimulation: optimization of stimulation and recording parameters. J. Neural Transm. 116, 1237-1242. doi: 10.1007/s00702-009-0 282-1

Randich, A., and Gebhart, G. (1992). Vagal afferent modulation of nociception. Brain. Res. Rev. 17, 77-99. doi: 10.1016/0165-0173(92)90009-B

Redgrave, J., Day, D., Leung, H., Laud, P. J., Ali, A., Lindert, R., et al. (2018). Safety and tolerability of transcutaneous vagus nerve stimulation in humans; a systematic review. Brain Stimul. 11, 1225-1238. doi: 10.1016/j.brs.2018. 08.010

Rong, P., Liu, A., Zhang, J., Wang, Y., Yang, A., Li, L., et al. (2014). An alternative therapy for drug-resistant epilepsy: transcutaneous auricular vagus nerve stimulation. Chin. Med. J. 127, 300-304. doi: 10.3760/cma.j.issn.0366-6999.20131511

Rong, P., Liu, J., Wang, L., Liu, R., Fang, J., Zhao, J., et al. (2016). Effect of transcutaneous auricular vagus nerve stimulation on major depressive disorder: a nonrandomized controlled pilot study. J. Affect. Disord. 195, 172-179. doi: 10.1016/j.jad.2016.02.031

Rong, P. J., Fang, J. L., Wang, L. P., Meng, H., Liu, J., ge Ma, Y., et al. (2012). Transcutaneous vagus nerve stimulation for the treatment of depression: a study protocol for a double blinded randomized clinical trial. $B M C$ Complement. Altern. Med. 12:255. doi: 10.1186/1472-6882-12-255
Safi, S., Ellrich, J., and Neuhuber, W. (2016). Myelinated axons in the auricular branch of the human vagus nerve. Anat. Rec. 299, 1184-1191. doi: 10.1002/ar.23391

Schulz-Stübner, S., and Kehl, F. (2011). Treatment of persistent hiccups with transcutaneous phrenic and vagal nerve stimulation. Intens. Care Med. 37, 1048-1049. doi: 10.1007/s00134-011-2150-3

Seiden, D., Corbett, S. A., and Lachman, E. (2013). Lachman's Case Studies in Anatomy. Oxford: Oxford University Press.

Sellaro, R., Steenbergen, L., Verkuil, B., van IJzendoorn, M. H., and Colzato, L. S. (2015a). Transcutaneous vagus nerve stimulation (tVNS) does not increase prosocial behavior in cyberball. Front. Psychol. 6:499. doi: 10.3389/fpsyg.2015.00499

Sellaro, R., Van Leusden, J. W. R., Tona, K.-D., Verkuil, B., Nieuwenhuis, S., and Colzato, L. S. (2015b). Transcutaneous vagus nerve stimulation enhances post-error slowing. J. Cogn. Neurosci. 27, 2126-2132. doi: 10.1162/jocn_a_0 0851

Shiozawa, P., da Silva, M. E., de Carvalho, T. C., Cordeiro, Q., Brunoni, A. R., and Fregni, F. (2014). Transcutaneous vagus and trigeminal nerve stimulation for neuropsychiatric disorders: a systematic review. Arquiv. Neuro Psiquiatr. 72, 542-547. doi: 10.1590/0004-282X20140061

Silberstein, S. D., Calhoun, A. H., Lipton, R. B., Grosberg, B. M., Cady, R. K., Dorlas, S., et al. (2016a). Chronic migraine headache prevention with noninvasive vagus nerve stimulation: the EVENT study. Neurology 87, 529538. doi: 10.1212/WNL.0000000000002918

Silberstein, S. D., Mechtler, L. L., Kudrow, D. B., Calhoun, A. H., McClure, C., Saper, J. R., et al. (2016b). Non-invasive vagus nerve stimulation for the acute treatment of cluster headache: findings from the randomized, double-blind, Sham-controlled ACT1 study. Headache 56, 1317-1332. doi: 10.1111/head. 12896

Standring, S. (2015). Gray's Anatomy: The Anatomical Basis of Clinical Practice. Philadelphia, PA: Elsevier Limited.

Stavrakis, S., Humphrey, M. B., Scherlag, B. J., Hu, Y., Jackman, W. M., Nakagawa, H., et al. (2015). Low-level transcutaneous electrical vagus nerve stimulation suppresses atrial fibrillation. J. Am. Coll. Cardiol. 65, 867-875. doi: 10.1016/j.jacc.2014. 12.026

Steenbergen, L., Sellaro, R., Stock, A.-K., Verkuil, B., Beste, C., and Colzato, L. S. (2015). Transcutaneous vagus nerve stimulation (tVNS) enhances response selection during action cascading processes. Eur. Neuropsychopharmacol. 25, 773-778. doi: 10.1016/j.euroneuro.2015. 03.015

Stefan, H., Kreiselmeyer, G., Kerling, F., Kurzbuch, K., Rauch, C., Heers, M., et al. (2012). Transcutaneous vagus nerve stimulation (t-VNS) in pharmacoresistant epilepsies: a proof of concept trial. Epilepsia 53, e115-e118. doi: 10.1111/j.1528-1167.2012.0 3492.x

Straube, A., Ellrich, J., Eren, O., Blum, B., and Ruscheweyh, R. (2015). Treatment of chronic migraine with transcutaneous stimulation of the auricular branch of the vagal nerve (auricular t-VNS): a randomized, monocentric clinical triial. $J$. Headache Pain 16:543. doi: 10.1186/s10194-015-0543-3

Tekdemir, I., Aslan, A., and Elhan, A. (1998). A clinico-anatomic study of the auricular branch of the vagus nerve and Arnold's ear-cough reflex. Surg. Radiol. Anat. 20, 253-257. doi: 10.1007/s00276-998-0 253-5

Trevizol, A. P., Shiozawa, P., Taiar, I., Soares, A., Gomes, J. S., Barros, M. D., et al. (2016). Transcutaneous vagus nerve stimulation (taVNS) for major depressive disorder: an open label proof-ofconcept trial. Brain Stimul. 9, 453-454. doi: 10.1016/j.brs.2016. 02.001

Van Leusden, J. W. R., Sellaro, R., and Colzato, L. S. (2015). Transcutaneous vagal nerve stimulation (tVNS): a new neuromodulation tool in healthy humans? Front. Psychol. 6:102. doi: 10.3389/fpsyg.2015.00102

Ventureyra, E. C. G. (2000). Transcutaneous vagus nerve stimulation for partial onset seizure therapy. Childs Nerv. Syst. 16, 101-102. doi: $10.1007 / \mathrm{s} 003810050021$

Vonck, K., De Herdt, V., and Boon, P. (2009). Vagal nerve stimulation-a 15-year survey of an established treatment modality in epilepsy surgery. Adv. Tech. Stand Neurosurg. 34, 111-146. doi: 10.1007/978-3-211-78741-0_5 
Walker, B. R., Easton, A., and Gale, K. (1999). Regulation of limbic motor seizures by GABA and glutamate transmission in nucleus tractus solitarius. Epilepsia 40, 1051-1057. doi: 10.1111/j.1528-1157.1999.tb00818.x

Weise, D., Adamidis, M., Pizzolato, F., Rumpf, J.-J., Fricke, C., and Classen, J. (2015). Assessment of brainstem function with auricular branch of vagus nerve stimulation in Parkinson's disease. PLoS ONE 10:e120786. doi: 10.1371 /journal.pone.0120786

Wexler, A. (2015). A pragmatic analysis of the regulation of consumer transcranial direct current stimulation (TDCS) devices in the United States: Table 1. J. Law Biosci. 2:lsv039. doi: 10.1093/jlb/lsv039

Yakunina, N., Kim, S. S., and Nam, E.-C. (2018). BOLD fMRI effects of transcutaneous vagus nerve stimulation in patients with chronic tinnitus. PLoS ONE 13:e0207281. doi: 10.1371/journal.pone.0207281

Yu, Y., Yang, Y., Wang, L., Fang, J., Chen, Y., He, J., et al. (2017). Transcutaneous auricular vagus nerve stimulation in disorders of consciousness monitored by fMRI: The first case report. Brain Stimul. 10, 328-330. doi: 10.1016/j.brs.2016.12.004

Yu, Z. J., Weller, R. A., Sandidge, K., and Weller, E. B. (2008). Vagus nerve stimulation: can it be used in adolescents or children with treatment-resistant depression? Curr. Psychiatry Rep. 10, 116-122. doi: 10.1007/s11920-008-0021-6

Yuan, H., and Silberstein, S. D. (2016a). Vagus nerve and vagus nerve stimulation, a comprehensive review: part I. Headache 56, 71-78. doi: 10.1111/head.12647
Yuan, H., and Silberstein, S. D. (2016b). Vagus nerve and vagus nerve stimulation, a comprehensive review: part II. Headache 56, 259-266. doi: 10.1111/head.12650

Zhao, B., Li, L., Jiao, Y., Luo, M., Xu, K., Hong, Y., et al. (2019). Transcutaneous auricular vagus nerve stimulation in treating post-stroke insomnia monitored by resting-state fMRI: The first case report. Brain Stimul. 12, 824-826. doi: 10.1016/j.brs.2019.02.016

Conflict of Interest: The authors declare that the research was conducted in the absence of any commercial or financial relationships that could be construed as a potential conflict of interest.

The Handling Editor declared a past co-authorship with one of the authors TK.

Copyright (c) 2020 Yap, Keatch, Lambert, Woods, Stoddart and Kameneva. This is an open-access article distributed under the terms of the Creative Commons Attribution License (CC BY). The use, distribution or reproduction in other forums is permitted, provided the original author(s) and the copyright owner(s) are credited and that the original publication in this journal is cited, in accordance with accepted academic practice. No use, distribution or reproduction is permitted which does not comply with these terms. 